\title{
A Survey on an Emerging Area: Deep Learning for Smart City Data
}

\author{
Qi Chen, Wei Wang, Fangyu Wu, Suparna De Member, IEEE, Ruili Wang Member, IEEE, Bailing Zhang, \\ and Xin Huang
}

\begin{abstract}
Rapid urbanisation has brought about great challenges to our daily lives, such as traffic congestion, environmental pollution, energy consumption, public safety and so on. Research on smart cities aims to address these issues with various technologies developed for the Internet of Things. Very recently, the research focus has shifted towards processing of massive amount of data continuously generated within a city environment, e.g., physical and participatory sensing data on traffic flow, air quality, and healthcare. Techniques from computational intelligence have been applied to process and analyse such data, and to extract useful knowledge that helps citizens better understand their surroundings and informs city authorities to provide better and more efficient public services. Deep learning, as a relatively new paradigm in computational intelligence, has attracted substantial attention of the research community and demonstrated greater potential over traditional techniques. This paper provides a survey of the latest research on the convergence of deep learning and smart city from two perspectives: while the technique-oriented review pays attention to the popular and extended deep learning models, the application-oriented review emphasises the representative application domains in smart cities. Our study showed that there are still many challenges ahead for this emerging area owing to the complex nature of deep learning and wide coverage of smart city applications. We pointed out a number of future directions related to deep learning efficiency, emergent deep learning paradigms, knowledge fusion and privacy preservation, and hope these would move the relevant research one step further in creating truly distributed intelligence for smart cities.
\end{abstract}

Index Terms-Deep learning, Smart city, Machine learning, Data Processing, Internet of things.

\section{INTRODUCTION AND BACKGROUND}

It is reported by the United Nations that $54 \%$ of the world population resided in urban areas in the year of 2014 and by 2050 the figure is expected to increase to $66 \%$ [1]. Continuous urbanisation poses severe challenges on sustainable development and living quality of urban residents. The vision of smart city is to make more efficient uses of scarce resources, and improve quality of citizen lives and public

Qi Chen, Fangyu Wu, Wei Wang, Bailing Zhang and Xin Huang are with the Department of Computer Science and Software Engineering, Xi'an Jiaotong Liverpool University, China. Xin Huang is also with Soochow University and Suzhou L.J.T Intelligent Technology Co., Ltd., Suzhou, China. E-mail: \{qi.chen,fangyu.wu,wei.wang03,bailing.zhang,xin.huang\}@xjtlu.edu.cn. Suparna De is with the Institute for Communication Systems (ICS), University of Surrey, UK. e-mail: s.de@surrey.ac.uk

Ruili Wang is with Institute of Natural and Mathematical Sciences, Massey University, Auckland, New Zealand. e-mail: Ruili.wang@massey.ac.nz Manuscript received xxx, 2018; revised . services [2]. With the development of Internet of Things (IoT), embedded devices, e.g., sensors, actuators, mobiles phones and Radio Frequency Identifications (RFIDs), can be built into every fabric of urban environments and connected with each other. Data generated by these devices can be preprocessed, integrated, and made available in standard formats through open services [3]. Numerous smart city applications have been developed and deployed, for example intelligent transportation [4], [5], [6], smart healthcare [7], [8], [9], environment monitoring [10], [11], [12], and public safety [13], [14], [15], just to name a few.

From a data-centric perspective, the key concept in smart cities lies in the sophisticated data analytics for understanding, monitoring, regulating and planning the city [16]. It is widely accepted that the process of smart city data analysis can be abstracted as four layers although different work may have some minor variations [5], [6], [12], [17]: data acquisition, data preprocessing, data analysis, and service provision, as shown in Figure 1: (1) the data acquisition layer is concerned about collecting and storing smart city data from various domains and sources; (2) the data preprocessing layer is responsible for preprocessing (e.g., data cleaning, selection and interpolation) to obtain data of higher quality before analytics, as smart city data of different modalities often contains noise, uncertainty and missing values; (3) the data analytics layer is to perform intelligent analysis using various machine learning techniques to discover previously unknown knowledge and insights specific to different applications, e.g., classification models can be used to recognise human activities and regression models to predict traffic flows; and (4) the service provision layer is to develop intelligent services and applications based on the outcome of data analytics, for example, with the patterns and events detected from traffic, pollution and weather sensory data analysis to provide better public services.

Techniques based on Computational Intelligence (CI), e.g., artificial neural networks, fuzzy systems, and evolutionary computation, have achieved remarkable results in modelling, learning, searching, and optimisation problems for smart city applications [18], [19], [20]. Deep learning, a relatively "young" learning paradigm in the CI family, has in fact its origin from Artificial Neural Networks (ANNs). It allows computational models that are composed of multiple processing layers to learn representations of data with multiple levels of abstraction, and is able to discover intri- 


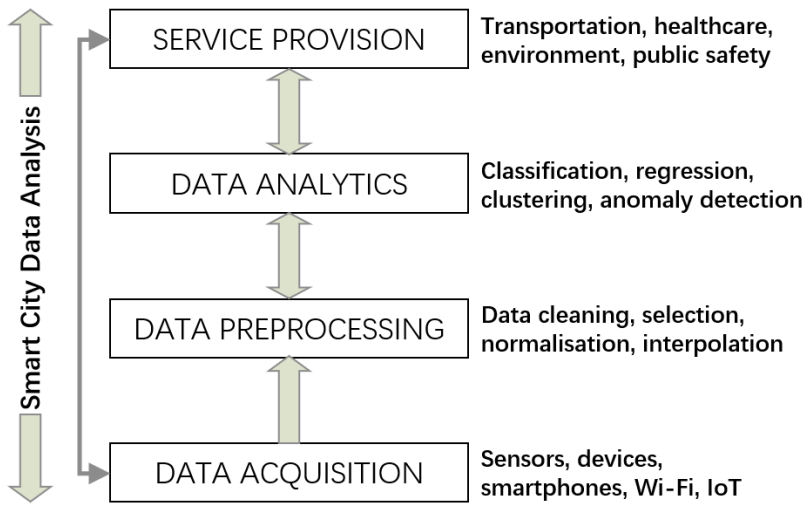

Fig. 1. A general framework for smart city data analysis.

cate structures from natural data in its raw forms without the needs of sophisticated feature engineering and tuning [21]. Compared to traditional machine learning methods, deep learning can model extremely sophisticated functions through layers of non-linear transformation trainable from the beginning to the end. Methods based on deep models have significantly improved the state-of-the-art in a wide array of problems, such as natural language processing (or more specifically, neural machine translation) [22], [23], [24], computer vision [25], [26], [27], and speech recognition [28], [29], [30].

The characteristics of deep learning make it attractive for analysing smart city data which has complex nature, i.e., different modalities (streaming, time-series, image, video and text), large amount (continuous data generated by millions of sensing devices), spatial and temporal dependency, etc. Researchers in smart cities have applied deep learning in many domains, e.g., traffic prediction, healthcare, air quality prediction and public safety. Although there have been surveys conducted in smart city data analysis [31], [32], [33] and deep learning [15], [34], we have not found any systematic studies on the convergence of these two areas. We survey the relevant research from two perspectives: in the technique-oriented review, we focus on the popular deep learning techniques as well as their variants and elicit their applications in different smart city domains; in the application-oriented review, we start with the representative application domains and discuss the different deep learning methods. While it is not possible to enumerate and review all of the studies in this emerging area, we wish to draw a clear landscape of the research on the intersection of deep learning and smart city data processing. We hope that the study will motivate more practical deep learning research and development toward more intelligent data analytics in smart cities.

The rest of the paper is organised as follows. In section 2 , we explain the data representation of different modalities: sensor stream data, image data and text data. In section 3, we first review some of the influential deep learning models developed in the past years and discuss their applications in smart city data analysis. In section 4 , we survey the studies in smart city applications related to transportation, health care, environment, and public safety, and investigate the development of deep learning in these areas. In section
5 , we present challenges, future directions, and concluding remarks.

\section{City Data Modalities and Representation}

City data can be collected from a large number of heterogeneous sources, e.g., sensor data from fixed and mobile sites as well as user-contributed data in participatory sensing [35]. From the many specific city data types as shown in Tables 1, 2, 3, 4 and 5, three representative data modalities can be observed: sensor data, image/video data and text data. These data modalities and their representations suitable for deep learning are described below.

\subsection{Sensor Data}

Different sensing devices have been deployed in many city applications, e.g., traditional physical sensors (fixed and mobile) have been widely used to measure physical phenomenon (e.g., temperature, air quality, light and traffic flow); wearable sensors and smartphones equipped with accelerometers, magnetometer, and gyroscopes, have been used to recognise human activities and behaviour. Sensors in different applications have very different sampling rates: while Electroencephalogram (EEG) sensors used in healthcare offer millisecond-range temporal resolution, air quality sensors report in minutes. Sensor measurement data is almost always associated with metadata, e.g., spatial information (where the measurement happens) and temporal information (when), which is important for sensor data representation and analysis.

Sensor Data Representation - There have been various representation formalisms proposed for sensor data, e.g., the Semantic Sensor Network (SSN) ontology [36], FUTS data model (Frequently Updated, Timestamped and Structured) [37], sensor stream representation [38] and the mobile sensor data model [39]. These representations focus on individual sensor observations and highlight the importance of modelling temporal and spatial information. With deep learning, input sensor data can be roughly represented as a temporal sequence of elements in the form $x=\left\{x_{1}, x_{2}, \ldots x_{t}, \ldots, x_{T}\right\}$, where $x$ denotes the sequence to be processed and $T$ denotes the length of $x$. Each $x_{t}$ in the sequence is a vector representing the data at time step $t$. Spatial information may be embedded in $x_{t}$ and its meaning is application dependent. For example, in traffic flow prediction [40], [41] and human activity recognition with smartphones [42], [43], $x$ represents a sequence of sensor values (traffic flow/speed and accelerometers/gyroscopes, respectively). In these applications, spatial information does not necessarily need to be considered as the sensors are installed at fixed locations. However, in processing GPS sensor data, spatial information has to be considered and can be embedded in $x$ through Geo-convolutional operations [44].

\subsection{Image and Video Data}

Image and video data have been pervasive in smart cities and can be collected in many ways: surveillance cameras are widely installed in city areas for public safety and criminal investigation; Unmanned Aerial Vehicles (UAVs) equipped with remotely controllable devices (i.e., sensors, cameras, 
and actuators) [45] are used for crowd surveillance [46] and disaster management [47]; satellite images are analysed to detect and classify objects on earth for environment protection and weather forecasting; citizens use smartphones to take images and videos to record daily lives and report social events.

Image and Video Data Representation - A digital image is usually represented as a three-dimensional matrix with numeric values. Each point in an image is called a pixel, denoting the colour value at that specific position. The number of rows and columns of an image is fixed and indicates the resolution of the image. To effectively characterise an image and make its representation robust to common variances, e.g, translation, illumination and occlusion, choice of features is crucial. Methods for image features can be roughly divided into two categories: hand-crafted and machine learned features. For hand-crafted features, one needs to manually design a feature extraction pipeline and algorithms based on expert knowledge. The most representative handcrafted features include Scale-Invariant Feature Transform (SIFT) [48] and Histogram of Oriented Gradients (HOG) [49]. In contrast, with deep learning, features can be extracted automatically from image datasets. For imagebased video representation, the standard method computes the representation by pooling all the descriptors from all the frames in a video. It can be summarised as: (1) obtain the descriptors for individual frames; (2) apply normalisation on frame descriptors; (3) perform average pooling on frame descriptors to obtain video representation, i.e., $x_{\text {video }}=\frac{1}{N} \sum_{i=1}^{N} x_{i}$, where $x_{i}$ is the frame-level descriptor and $N$ is the total number of frames extracted from the video; and (4) apply re-normalisation on the video representation.

\subsection{Text Data}

As smartphones and wireless networks become pervasive, the idea of 'citizen sensing' has been proposed to describe the activities of network of people who actively observe, collect, analyse, report and disseminate information through text, audio, or video [50]. While data produced by the citizen sensors differs semantically from sensing data generated by the physical devices to a great extent, it is a necessary complementary data source for many smart city applications. One of the typical applications is to analyse short textual messages posted on social media platforms (e.g., Twitter) to discover knowledge from multiple perspectives in real time, e.g., social event detection in Twitris [51], traffic event detection [52], [53], disease activity tracking [54], and natural disease monitoring [55], [56]. One key advantage of making use of social textual data is that the discovered knowledge has a higher level of semantics which is more intuitive for human beings to comprehend.

Text Data Representation - Processing textual data heavily relies on natural language processing techniques. In deep learning, textual data can be represented as temporal sequence of variable lengths, similar to sensor data representation, i.e., $x=\left\{x_{1}, x_{2}, \ldots x_{t}, \ldots, x_{T}\right\}$, where $x$ denotes a sentence or text block and $x_{t}$ a vector representing a word at time step $t$. Two common word representation methods are the one-hot representation and word embedding [57], [58].
One-hot representation of a word sets the corresponding element as ' 1 ' (' 0 ' for all others) in a vector whose dimensionality equals to the size of the vocabulary. The representation is simple and not able to capture relational structure of the lexicon. The word embedding representation has become the norm for natural language processing tasks using deep learning. It involves a mathematical embedding from a space with one dimension per word to a continuous vector space with a much lower dimension. The resultant embedding has a key characteristic that similar words end up having values closer to each other although each word is assigned with a different vector [57], [58].

\section{Deep Learning Oriented Perspective}

In this section, we first give a general overview about deep learning, and then we discuss in detail how different deep learning models are applied in smart city data analytics. We focus on the four most widely used deep neural network models: Recurrent Neural Network (RNN) [59], Convolutional Neural Network (CNN) [60], Deep Belief Networks (DBN) [61], and Stacked AutoEncoder (SAE) [62].

\subsection{Overview of Deep Learning}

Deep learning originated from the study of Artificial Neural Networks (ANNs), which are computation models inspired by biological neural networks in human brains and have been extensively studied since the 1980s. An ANN consists of a collection of connected artificial neurons which simulate the neurons in a biological brain. It can be roughly characterised by the weights between layers of neurons whose output is computed based on some non-linear transformation function. At each layer, neurons compute a weighted sum of the inputs from the previous layer, using $W x+b$ where $W$ is a weight vector and $b$ is a bias, and pass the result through a non-linear activation function $\sigma$, e.g., tanh, sigmoid and rectified linear unit (ReLU) [63].

One of the major reasons that ANNs with multiple fully connected layers have not gained popularity in many realworld applications for decades is their computation complexity. The idea of deep learning, also inspired by biological processes, powered by high performance computing hardware, has made very deep models computationally feasible for real-world applications. For example, in a convolutional network, the connectivity between neurons resembles the organisation of neurons in animal visual cortex [21]. Each cortical neuron only responds to stimuli within a limited region of a visual field (also known as the receptive field); in a recurrent network, weights are shared among layers which not only reduces the number of parameters to be learned but also generalises better for input sequences of different lengths [21]. Training deep neural networks is notoriously expensive and would not be practical without the employment of high performance computing hardware, e.g., Graphics Processing Units (GPUs). The highly parallel structure ensures efficient processing of large number of data blocks in parallel, which makes it suitable for training deep neural networks that have thousands of neurons performing the same computation at each layer.

In recent years, deep learning methods have achieved results superior to other state-of-the-art machine learning 
methods and even human experts in many applications areas. We focus on the four most popular deep learning architectures, particularly in the field of smart city research: RNN, CNN, DBN, SAE, and various extended models as shown in Figure 2a. After introducing the technical detail of each models, we discuss the corresponding applications that make use of them.

\subsubsection{Training}

In deep learning, each layer transforms the input data into a more abstract representation and the model learns to choose the best features that can improve performance. It can be used for both supervised learning and unsupervised learning tasks. In supervised learning, the objective is usually to learn a complex, non-linear function that maps the input to the output. This requires learning algorithms to generalise from the training data to unseen data in a"reasonable" way. An objective function is normally used to measure the error (or distance) between the predicted output and the desired output. A common objective function for classification tasks is Cross Entropy (CE) as shown in Equation 1, where $x$ is an instance in the training set, $p(x)$ is the true probability distribution of the dependent variable while $q(x)$ is the predicted probability distribution.

$$
C E=-\sum_{x} p(x) \log q(x)
$$

A common objective function for regression tasks is Mean Squared Error (MSE), as defined in Equation 2, where $|X|$ is the size of the training set, $y$ is the ground truth and $\hat{y}$ is the network output for regression problem

$$
M S E=\frac{1}{|X|} \sum_{x}(y(x)-\hat{y}(x))^{2}
$$

Some deep learning models can also be trained in unsupervised ways in which the output tries to recover the input and the objective is to minimise the reconstruction. For example, the work in [61] showed that deep belief networks can be trained in an unsupervised manner (pre-training), followed by a supervised fine-tuning, which resulted in superior performance.

After determining objective function, deep models are then trained to minimise the objective function with backpropagation and gradient descent techniques. Backpropagation algorithm distributes the error computed at the output layer backwards and the gradients of weights at different layers are calculated. At each layer, the gradient descent algorithm computes a gradient vector, and adjusts the weight vector along the opposite direction of the gradient vector to minimise the objective function. In practice, gradientbased learning algorithms, e.g., Stochastic Gradient Descent (SGD) [64], Adam [65] and RMSprop [66], have been widely adopted together with backpropagation for neural network training.

\subsubsection{Regularisation}

One of the common goals of machine learning algorithms is to generalise to unseen data. Overfitting happens when a model learns too well the details and the noise from training data while ignoring the general patterns, thus results in poor generalisation. Regularisation is a critical instrument in preventing overfitting. Some of the most common regularisation techniques for deep learning are: dataset augmentation, L1 and L2 regularisation, early stopping, and dropout, as suggested in [67].

Dataset augmentation - overfitting can be a common problem when size of training data is too small compared with the number of model parameters to be learned. While an existing dataset may be limited, for some applications one may create synthetic data through a number of operations, e.g., rotate, scale and inject random unrelated images to enlarge a dataset. Besides creating synthetic data, multi-task learning and transfer learning techniques are also commonly used. In multi-task learning, related tasks using different datasets can be learned simultaneously. The work in [17], [68], [69] applied multi-task learning to jointly learn people's movement and transportation mode patterns. Often when a training dataset may be too specific or small to learn a good model from scratch, transfer learning can be applied by pre-training a model with large available dataset and then fine-tuning the model with the data for specific tasks such as fire detection [70], parking lot detection [71], and crisis-related tweets classification [56].

L1 and L2 regularisation - to prevent model from becoming too complex (e.g., large weights) and learning all the details and noise in the training dataset, a regularisation term can be added to the objective function. L1 regularisation (or Lasso regression) and L2 regularisation (or Ridge regression) are commonly used not only in deep learning but also many other machine learning algorithms. L1 regularisation is defined using absolute values of the weights and can perform some sort of feature selection, while $\mathrm{L} 2$ regularisation is defined using the squared values of the weights to penalise large model parameters.

Early stopping - in an ideal situation, as a model sees more data both training and test errors should constantly decrease. However, after certain number of epochs, the model may start to overfit and learn noise in the training set. In this case, the training error keeps going down while the test error starts to increase. Early stopping is used here to find the right moment to stop training to minimise the test error.

Dropout - it refers to a strategy that randomly drops out some units (hidden and visible) in a deep neural network to make nodes become more insensitive to the weights of the other nodes. It provides a way of approximately combining many different neural network architectures efficiently [72].

\subsection{Recurrent Neural Networks (RNN)}

A RNN [59] contains links among neurons, and after unfolding it forms a directed graph along a sequence. This allows RNN to process data that can be modelled as temporal sequences of variable lengths, $x=\left(x_{1}, \ldots, x_{T}\right)$. At each time step $t$, the hidden state $h_{t}$ of the RNN is updated using $h_{t}=f\left(h_{t-1}, x_{t}\right)$, where $f$ is a non-linear function, which can be as simple as an sigmoid function and as complex as a long short-term memory unit. RNNs use the internal states to capture dependency among input data in a sequence, which makes them suitable to tasks such as natural language processing, speech recognition and smart 


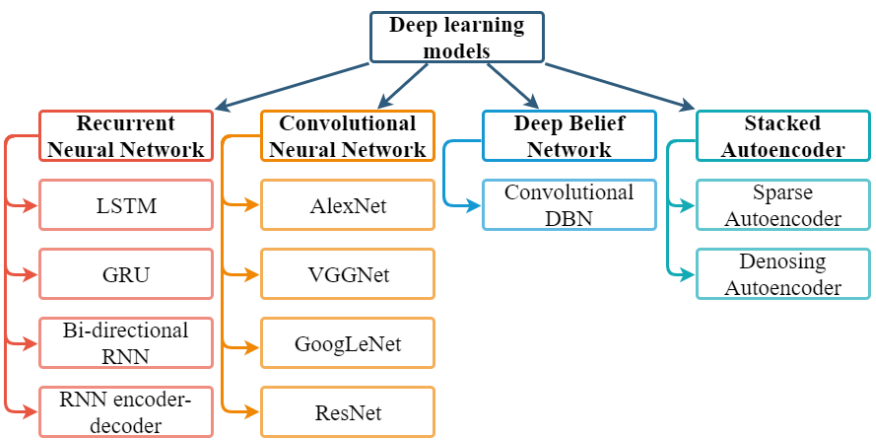

(a) technical perspective

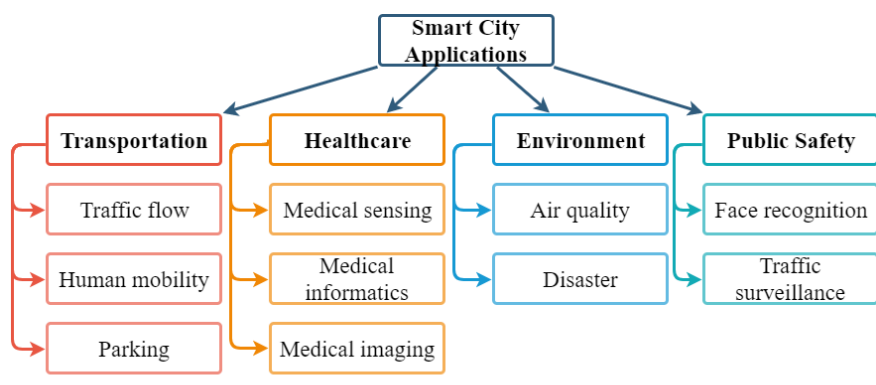

(b) application perspective

Fig. 2. Classification of the deep learning research in smart city data analytics from two different perspectives.

city applications in which data demonstrates strong temporal correlations. As vanilla RNNs suffers from various limitations, they have not been used in any real-world applications. In what follows, we present two important RNN units: Long Short-Term Memory (LSTM) and Gated Recurrent Unit (GRU), and two widely used architectures: Bi-directional RNN and RNN encoder-decoder; and then discuss their usage in different smart city applications.

\subsubsection{Long Short-Term Memory}

Vanilla RNNs have difficulties in modelling long sequences as the gradients in parameter updates tend to either explode or vanish during backpropagation. LSTM has been proposed to solve the vanishing and exploding gradient problem by introducing the idea of Constant Error Carousels (CEC) [73]. In the original LSTM, the activation function of the unit is replaced by the identity function in the CEC to enforce constant error flow. Later, various extended models have been proposed, e.g., by adding forget gate and peephole connection [29] in order to address the limitations of the original LSTM [73].

We briefly introduce the LSTM unit, following the notations used in [29], [74]. As shown in Figure 3a, it contains a cell $C$, an input gate $i$, an output gate $o$ and a forget gate $f$. The subscript $t$ represents a particular time step. A standard LSTM updates the hidden state $h$ by iterating the following steps shown in Equation 3, where all the $W$ and $U$ matrices are the learnable weights and the $b$ vector represents the bias term (We ignore the subscripts for simplicity).

$$
\begin{array}{r}
f_{t}=\sigma\left(W_{f} x_{t}+U_{f} h_{t-1}+b_{f}\right) \\
i_{t}=\sigma\left(W_{i} x_{t}+U_{i} h_{t-1}+b_{i}\right) \\
C_{t}=f_{t} * C_{t-1}+i_{t} * \tanh \left(W_{C} x_{t}+U_{C} h_{t-1}+b_{C}\right) \\
o_{t}=\sigma\left(W_{o} x_{t}+U_{o} h_{t-1}+b_{o}\right) \\
h_{t}=o_{t} * \tanh \left(C_{t}\right)
\end{array}
$$

The standard LSTM based RNNs have been applied in many smart city applications, e.g., traffic prediction [40], [41], healthcare analysis [8] and air quality prediction [10]. Taking traffic prediction as an example, the task is to predict traffic flow $x_{T+1}^{i}$ at time step $T+1$ based on the historical traffic flow sequence $X=\left\{x_{t}^{i} \mid i=1,2, \ldots, n, t=1,2, \ldots, T\right\}$, where $n$ denotes the number of traffic observation stations. At each time step, the traffic flow vector $x_{t}^{i}$ is fed into the corresponding input layer. After training, the network learns the relationship of different stations and time steps, and uses the last $h_{t}$ to predict the traffic flow of all stations at time $T+1$.

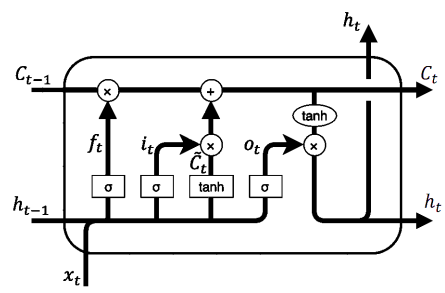

(a) Long Short-Term Memory

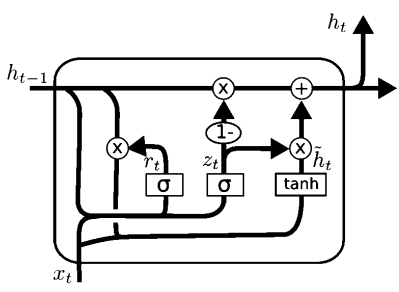

(b) Gated Recurrent Unit
Fig. 3. Two widely used recurrent units in RNNs. Diagrams adapted from [74]

\subsubsection{Gated Recurrent Unit}

Gated Recurrent Unit (GRU), as shown in Figure 3b, is another RNN unit introduced by Cho et al. [23]. It contains two gates: update gate $z$ and reset gate $r$, where the update gate helps the model determine how much of the past information to remember and the reset gate is used to decide how much of the past information to forget. The hidden state $h$ is then updated iteratively using the following procedure shown in Equation 4, where all the $W$ and $U$ matrices are the learnable weights and the $b$ vector represents the bias terms.

$$
\begin{array}{r}
z_{t}=\sigma\left(W_{z} x_{t}+U_{z} h_{t-1}+b_{z}\right) \\
r_{t}=\sigma\left(W_{r} x_{t}+U_{r} h_{t-1}+b_{r}\right) \\
h_{t}=\left(1-z_{t}\right) * h_{t-1}+z_{t} * \sigma_{h}\left(W_{h} x_{t}+\right. \\
\left.U_{h}\left(r_{t} * h_{t-1}\right)+b_{h}\right)
\end{array}
$$

The GRU unit is simpler and more efficient than the standard LSTM. It has become increasingly popular in natural language processing [23], speech recognition [30], traffic prediction [75] and air quality prediction [76].

\subsubsection{Bi-directional RNN}

A standard RNN can learn representations from data from previous time steps; however, representations from future 
time steps may help better understand the context and eliminate ambiguity. For example, in handwriting recognition, the performance can be significantly enhanced if the letters located before and after the current letter were known. Bi-directional RNNs [77] was proposed by stacking two LSTM RNNs, one processing the sequence from left to right, the other one from the opposite direction, and finally concatenating the output of the two RNNs. With this structure, the output layer can integrate information from both past and future states.

Bi-directional RNNs have been commonly used in natural language processing [78] and speech recognition [29]. For sensor data processing, recent studies applied Bi-directional LSTM to recognise human activities [43], [79]. In this task, the input is a discrete sequence of equally spaced samples $\left\{x_{1}, x_{2}, \ldots, x_{t}\right\}$, where each data point $x_{t}$ is a vector of individual samples observed by sensors at time $t$. The samples are segmented into windows of maximum time $T$ and fed into the network with one direction from time 1 to $T$ and another direction from time $T$ to 1 . The network can output the probabilities of different activity labels after a softmax layer. Both work [43], [79] reported the state-ofthe-art performance compared to conventional techniques.

\subsubsection{RNN Encoder-Decoder}

In some applications, the input and output sequences have different lengths, e.g., in machine translation, the input sentence and the desired target sentence usually have different lengths. An important and effective technique for such application is the RNN based encoder-decoder architecture [23]. It contains two RNNs, one learns to encode an input sequence of certain length into a context vector representation (the encoder) and the other learns to decode the context vector representation back into an output sequence of different length (the decoder).

The architecture allows some smart city applications to produce a sequence of predictions for time series data. For example, in air quality and water quality prediction task, the work in [80] designed an encoder to find a suitable representation of the past observation data and used a decoder to generate a sequence of output, i.e., the air and water quality measurement in the next few minutes or even hours. In addition, the work applied spatial attention in the input layer and embed some external factors, e.g., time, weather and point of interests, in the context vector to further improve prediction results.

\subsection{Convolutional Neural Networks (CNN)}

A typical CNN [60] usually consists of a number of convolutional layers, pooling layers, and fully connected layers as its hidden layers as illustrated in Figure 4. The convolutional layer aims to learn filters that represent features of the input (e.g., a particular shape) and generate a feature map. The pooling layer performs non-linear down sampling, which combines a cluster of neurons at one layer into a single neuron in the next based on non-linear functions such as max pooling and average pooling. Then, fully connected layers are added on the top of convolutional and pooling layers for final output. In Figure 4, the output is represented by $p(y \mid x)$, which stands for the probability distribution over the dependent variable $y$, given an input $x$. In recent years, CNN has achieved superior results in image and video recognition [27], speech recognition [81], and natural language processing [82].

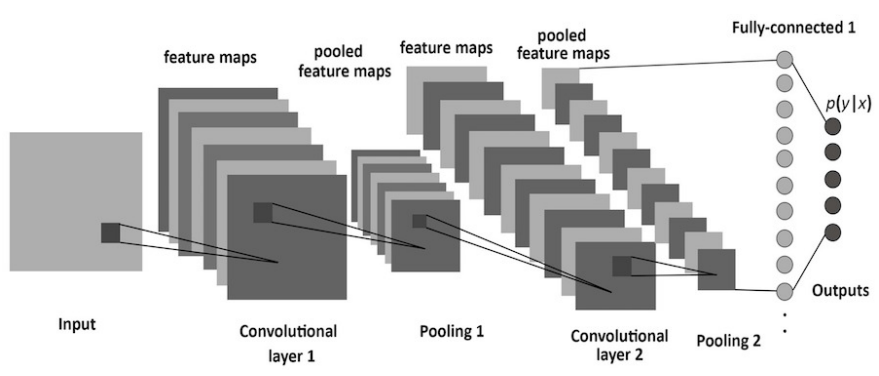

Fig. 4. A typical Convolutional Neural Network architecture. Figure adapted from [60]

In smart city research, $\mathrm{CNN}$ has been applied in applications related to healthcare, public safety and transportation. In processing sensor data, $\mathrm{CNN}$ can make use of spatially correlated sensor signals for tasks such as human mobility [6] and human activity recognition [42]. As CNN is primarily designed for processing images and videos, input adaptation, which transforms the sensor data to virtual images, is necessary. The study in [6] first partitioned a city into regions and then calculated the inflow and outflow of crowds in each region based on data collected from GPS trajectories and bike trips. Each region represents a pixel in a virtual image; and inflow and outflow of crowds represent the value for that pixel. For human activity recognition tasks, most sensors produce multi-dimensional 1D readings such as acceleration signal. There are mainly two types of transformation: the first is to treat each dimension as a channel, and perform 1D convolution, and the second is to combine readings from multiple sensors to form a virtual 2D image and then perform 2D convolution [42].

For image and video recognition tasks, many configurations and variants have been proposed in the literature for smart city applications. In [83], a random CNN was proposed to solve the vehicle license plate recognition problem. It has four layers implementing filter bank convolution, rectified-linear activation, spatial pooling, and divisive normalisation, respectively. The final architecture was learned from thousands of random possibilities. CNN has also been applied to provide robust face descriptors, and achieved state-of-art accuracy in face verification and recognition tasks [84], [85].

Recently, some notable variants of the CNN for image classification tasks, e.g., AlexNet [25], VGG [26], GoogLeNet [86] and ResNet [27], have been developed. When use these large scale networks for smart city applications, e.g., parking lot detection [87], fire detection [70], human mobility [6] and vehicle detection [13], a model pre-trained with large scale image datasets and fine-tuned with dataset in particular domain is usually preferred. This is also referred to as transfer learning, which needs further exploration for smart city applications. 


\subsection{Deep Belief Network (DBN)}

A DBN [61] is a generative graphical model which can learn deep representation from given data. It models the joint distribution between observed data and the hidden layers. A DBN can be viewed as a stack of layers of simple, unsupervised networks such as the Restricted Boltzmann Machine (RBM), which is an energy-based generative model with an input layer, a hidden layer and symmetric connections between them. In such a setting, the current hidden layer of RBM serves as the input layer for the next RBM. The whole network can also be fine-tuned with the backpropagation algorithm. This composition leads to a fast, layer-by-layer unsupervised architecture, which has become one of the very effective deep learning algorithms [61].

One of the notable characteristics of DBN is the capability of reconstructing the input in an unsupervised manner. As such, it has been applied to perform efficient unsupervised feature learning in the areas of transportation [17], [69] and healthcare [88]. For example, the work in [88] applied DBN to EEG Waveforms for anomaly detection. The idea is that when reconstructing the input, DBN tends to learn poorly for anomalous samples, which usually results in high reconstruction error. Therefore, abnormal EEG signals can be effectively detected by setting an error threshold.

As the standard DBN does not scale well to highdimensional data such as images, Convolutional Deep Belief Network (CDBN) composed of multiple layers of convolutional restricted Boltzmann machines was introduced in [89]. In CDBN, probabilistic max-pooling was applied to reduce the dimensions in higher layers in the network, which makes it efficient and effective for learning. The research in [90] applied CDBN to learn features from highdimensional and multichannel Electroencephalogram (EEG) data and reported that the learned features outperformed other state-of-the-art feature extraction methods.

\subsection{Stacked Autoencoder Network}

An Autoencoder (AE) attempts to reproduce its input by learning a set of representations in an unsupervised manner. More formally, given a training instance $x$, where $x \in \mathbf{R}^{d}$, an AE first maps it to a latent representation $z$ by Equation 5 , where $\sigma$ is an activation function, $W$ is a weight matrix, and $b$ is bias vector. Then it tries to reproduce $x$ by decoding $\mathrm{z}$ to $\hat{x}$ which is of the same shape as $x$ by Equation 5 .

$$
\begin{array}{r}
z=\sigma(W x+b) \\
\hat{x}=\sigma^{\prime}\left(W^{\prime} z+b^{\prime}\right)
\end{array}
$$

Autoencoders are trained to adjust the weights by minimising reconstruction errors $L(x, \hat{x})$. Equation 6 defines the function using squared errors:

$$
L(x, \hat{x})=\|x-\hat{x}\|^{2}=\left\|x-\sigma^{\prime}\left(W^{\prime}(\sigma(W x+b))+b^{\prime}\right)\right\|^{2}
$$

Stacked autoencoder (SAE) [62] is formed by stacking each hidden layer of an $\mathrm{AE}$ on the top of others. By pretraining a number of hidden layers, the weights of a deep network could be initialised to sensible values from which more complex while effective representations can be learned progressively. A final output layer can be added to the top of the network and the whole deep network could be fine-tuned using the backpropagation algorithm. Similar to DBN, SAE is a powerful tool for automatic feature extraction in an unsupervised fashion. For small datasets, the unsupervised pre-training helps prevent overfitting which leads to better generalisation [91]. Two commonly used variations of $\mathrm{AE}$ are sparse autoencoder and denoising autoencoder.

\subsubsection{Sparse Autoencoder}

When the number of hidden units is larger than inputs, an AE may potentially learn the identity function. To address this issue, Sparse AE [92] was introduced, in which more hidden units than inputs can be added, but only a small number of the hidden units is allowed to be activated at the same time by imposing a sparsity constraint on the hidden units. The sparsity constraint improves the ability of the hidden units to learn better representations. Recent studies have applied sparse AEs to traffic prediction [93] and air quality prediction [12].

\subsubsection{Denoising autoencoder}

Even when an input is partially corrupted, a denoising AE, proposed in [94], can be applied to obtain a robust representation and recover the original undistorted input. To train a denoising $\mathrm{AE}$, the initial input is assumed to be corrupted, $x \rightarrow x^{\prime}$; the corrupted input $x^{\prime}$ is used for training a normal autoencoder. However the loss is computed based on the original input and the output $L\left(x, \hat{x}^{\prime}\right)$. As corrupted or missing data in a city environment is common, researchers have applied Stacked Denoising Autoencoder (SDAE) to various smart city applications related to healthcare [9], environment and transportation [95]. For example, in traffic data imputation task, a corrupted version of the traffic data was used for training and the reconstruction error was measured between the reconstructed traffic data and the original one. Therefore, the well trained DAE was able to learn a more robust representation of traffic data and recover the corresponding traffic data even though the input was corrupted [95].

\subsection{Hybrid model}

Hybrid model is referred to as the combination of more than one type of deep learning models. Recent research has seen a number of such hybrid models applied in different application domains in smart cities, e.g., $\mathrm{RNN}+\mathrm{CNN}$ for city transportation (travel time estimation) [44], healthcare (hand gesture recognition) [96] and public safety (license plate recognition) [97]; SAE + RNN for environment monitoring (PM2.5) [10]; and RNN + RBM for transportation (traffic congestion) [98], and etc.

One particularly interesting hybrid model is the combination of CNN and RNN, which enhances the capabilities of the deep learning model in processing data that exhibits strong spatial and temporal correlations. Such data in fact is very common in many smart city applications. In [44], the authors proposed a geo-convolution component to integrate geographic information into the classical convolution to capture the spatial dependency of taxi trajectories. A recurrent layer was then added on the top of the geo-convolution to capture temporal dependencies among the trajectories for 
accurate travel time estimation. In [96], a 3D-CNN was used to extract local spatial-temporal features from a video. Then, these features were fed into to a recurrent network for global temporal modelling for dynamic hand gestures detection, which cannot be easily realised with a standalone $\mathrm{CNN}$ or RNN. The work also reported that the model outperformed competing state-of-the-art algorithms based on evaluation with several standard benchmark datasets.

\subsection{Model Selection for Smart City Data}

Deep learning techniques are able to discover natural structures from raw data without the needs for complex and time-consuming feature engineering as required in most of the conventional learning paradigms. They perform direct end-to-end mapping from raw data input to output in different smart city applications. It is obvious that one deep model can be applied to solve different problems in the smart city domain, and one specific problem can be addressed by several deep learning models or combination of them. In general, the major advantage of using unsupervised pretraining (e.g., SAE and DBN) is that it can help prevent overfitting which often leads to better representation and generalisation, especially for small sized datasets [91]. However, when large amount of data is available, CNN and RNN demonstrate better prediction performance over SAE and DBN.

One of the questions to be asked is how to select the best model for a given city data type. To answer the question, we summarise the applications of different deep models, together with the input representation and the representative references in Table 1. Today, CNN has been the dominate model in processing data with strong spatial correlation, e.g., images used in medical research [99], [100], [101] and public surveillance [13], [104], as well as geographic location data used in human mobility [42]. RNN has been the first choice for modelling sequence data with strong temporal dependencies, e.g., sensor time series of traffic flow or speed [40], [41], [75], air quality [10], [76], [80], human activity [43], [79], etc. This can be roughly used as a first guideline in selecting an appropriate model. Nevertheless, there have been studies showing that $\mathrm{CNN}$ could also model temporal dependency in human mobility recognition [6] and short text classification [56].

Many types of city data demonstrate spatio-temporal correlations, e.g., GPS trajectories, videos, and meteorology data. Hybrid models that combine CNN with RNN [44], [96], [107] (or with some spatial temporal transformation or embedding mechanisms) work well and tend to perform better than any single models. Nevertheless, technically, it is difficult to make a straightforward conclusion on what the best model is for a particular problem. There are at least two reasons for this: (1) the lack of large-scale experiments and benchmark studies. Our study showed that in the experiments in most of the research work, the results were only compared to those generated by either conventional machine learning techniques or deep models falling in the same category with some parameter changes or minor architecture modifications. We have not found any notable studies performing horizontal comparison, i.e., evaluation against deep models in other categories; and
(2) the inherent complex nature of deep learning. Usually, there are extremely large number of parameters to train and performance of a model is sometimes very sensitive to parameter settings. Such a tedious process often prevents one from finding the best model and settings for a specific task. We expect to see that more benchmark datasets will be shared by the research community for more comprehensive evaluation studies.

\section{Application Oriented Perspective}

Smart city is a multi-disciplinary research field by its own nature and many of its applications have close connections with other long-existing research areas such as transportation, mobile computing and healthcare. An important reason that such applications are often described as being "intelligent" or "smart" is that they apply artificial intelligence techniques to perform sophisticated data analytics to extract meaningful and useful insights and understand the cities better.

Conventional techniques used in smart city applications have at least three major limitations: (1) they usually need a complex and time-consuming feature engineering process before perform data analysis. This has been reported in many existing studies, e.g., parking space detection [108], medical image analysis [109] and face recognition [110]; (2) conventional techniques with shallow architectures, e.g., ANN with one or few hidden layers, may not be able to learn effective representations, which often results in poor performance. Research in different domains, e.g., traffic flow prediction [111], medical diagnosis [112] and air quality prediction [113], has pointed out the same issue; (3) they cannot directly process raw smart city data which are often noisy and contain many missing values. They only work with high-quality datasets and cannot generalise well with corrupted or missing data as shown in [15], [114], [115]. In contrast, deep learning models can learn a robust representation from corrupted input, e.g. corrupted sensor data [95], incomplete electronic health records [116] and masked face images [117].

As reviewing an exhausted list of these applications is neither possible nor significative, we only study four representative application domains for which deep learning methods have been applied and demonstrated notable performance improvement, i.e., Transportation, Healthcare, Environment and Public safety. A classification of these application areas is shown in Figure 2b. Information about the nature of the studies, datasets used and methods is summarised in Tables 2, 3, 4, and 5, respectively. The "Remarks" columns in the tables highlight the most distinctive features of each individual studies.

\subsection{Transportation}

With deep learning, intelligence transportation systems are able to discover knowledge from traffic data and enable users make safer, more coordinated, and smarter use of the transport networks. We focus on three applications: transport flow, human mobility, and parking applications. Features of the selected studies are highlighted in Table 2. 


\begin{tabular}{|c|c|c|c|}
\hline Model & Input representation & City data & Reference \\
\hline \multirow{4}{*}{ RNN } & \multirow{4}{*}{ Time sequence data $\left\{x_{1}, x_{2}, \ldots, x_{t}\right\}$} & traffic flow or speed (sensor) & {$[40],[41],[75]$} \\
\hline & & air quality data (sensor) & {$[10],[76],[80]$} \\
\hline & & healthcare data (sensor) & {$[8]$} \\
\hline & & human activity (sensor) & [43], [79] \\
\hline \multirow{8}{*}{ CNN } & \multirow{8}{*}{$2 \mathrm{D}$ or 3D matrix } & human mobility (sensor) & [42] \\
\hline & & human activity (sensor) & [6] \\
\hline & & parking lot image & {$[71],[87]$} \\
\hline & & medical image & [99], [100], [101] \\
\hline & & disaster image & [47], [70] \\
\hline & & twitter data (text) & [56] \\
\hline & & face image & [102], [103] \\
\hline & & vehicle image & [13], [104] \\
\hline \multirow{2}{*}{ DBN } & \multirow{2}{*}{ vector $\left[x_{1}, x_{2}, \ldots, x_{n}\right]$} & traffic flow (sensor) & [17], [69] \\
\hline & & EEG signal (sensor) & [88], [89] \\
\hline \multirow{4}{*}{ SAE } & \multirow{4}{*}{ vector $\left[x_{1}, x_{2}, \ldots, x_{n}\right]$} & traffic flow (sensor) & [93], [95] \\
\hline & & air quality data (sensor) & [12] \\
\hline & & medical image & [105] \\
\hline & & sensing image & [106] \\
\hline \multirow{3}{*}{$\mathrm{CNN}+\mathrm{RNN}$} & \multirow{3}{*}{ Sequence of $2 \mathrm{D}$ or $3 \mathrm{D}$ matrices } & taxi trajectories (sensor) & {$[44]$} \\
\hline & & hand gesture video & [96] \\
\hline & & meteorology data (sensor) & [107] \\
\hline
\end{tabular}

Overview of deep learning models for different type of city data.

\subsubsection{Transport flow}

Road traffic information is needed by individual travellers, business sectors, and government agencies to make better travel decisions, alleviate traffic congestion, and improve traffic operation efficiency [121]. Loop sensors have been widely embedded in pairs in major roads (e.g., highways) to collect traffic data for transportation analysis, i.e., detecting traffic flow/speed by counting the number of traversing vehicles and the time interval that vehicles travel across a pair of sensors. Data collected from different roads can be used in combination to predict traffic flow/speed, analyse traffic congestion, and make better urban transportation planning.

A number of conventional models have been used for traffic flow prediction in the literature, e.g., ARIMA, $\mathrm{k}$ Nearest Neighbour (k-NN) [118], Support Vector Regression (SVR) [119] and ANN [111]. For time-series based methods like ARIMA, a linear method is often used and the future traffic flow is only predicted based on historical traffic flows on a particular road. The methods ignore the fact that transportation system is a highly correlated network. For k-NN and SVR, a complex feature engineering is mostly needed, which requires prior knowledge of the transportation domain. Also without proper pre-processing and cleaning, they cannot easily work with missing data. As mentioned earlier, ANN with shallow architectures often results in poor performance.

As the loop sensors are deployed at fixed locations, spatial information is usually not used in traffic flow and speed prediction. It is natural to see that only RNN, SAE and DBN have been used in existing research. The work of Huang et al. [69] was the first study to apply deep learning for the traffic flow prediction problem by using DBN to learn effective features in an unsupervised fashion. In addition, it added a multitask regression layer on the top of DBN for supervised prediction and reported around $5 \%$ improvements over the state of the art. As traffic is usually affected by other factors such as weather condition, the work in [17] developed a DBN based deep learning model to fuse traffic data and weather data for more accurate prediction. The studies in both [5] and [93] applied SAE for traffic flow prediction. While the work in [5] used a sparse AE for better feature extraction, the one in [93] used the Levenberg-Marquardt algorithm to train SAE for more stable convergence. As SAE and DBN cannot model temporal dependency in the data, RNN has also been used for traffic flow/speed prediction [40], [41]. Both work used LSTM based RNN for short-term traffic flow/speed prediction and reported better performance over other deep learning models.

\subsubsection{Human Mobility}

Location acquisition technologies like GPS and WiFi enable people to record a sequence of timestamped location history called trajectories. The trajectory data has been used for individual movement prediction or urban transportation modelling. However, most of studies only focused on single or multiple road segments, rather than the dynamics of human mobility on a city-wide scale [68], [6]. Recently, deep learning research made use of trajectory data for many purposes, e.g., human mobility prediction, taxi demand analysis, and estimate travel time.

Predicting human mobility in a city is of great importance for traffic management and public safety [31]. The work in [68] described an intelligent transportation system for simulating and predicting human mobility and transportation mode at a citywide level. The study collected GPS records of about 1.6 million anonymous users throughout Japan from 2010 to 2013, and transportation network data (i.e., road structures and point of interests information) of many cities in Japan. It applied LSTM based RNN with a multi-task learning architecture to jointly learn human mobility and transportation mode. It was used to predict future movement of citizens and their transportation modes in a large-scale transportation network. The work in [6] proposed the use of ResNet for crowd flow prediction. It first partitioned a city into regions based on the longitude and latitude, and then applied ResNet to forecast inflow and 


\begin{tabular}{|c|c|c|c|c|}
\hline Reference & Application & Data & Method & Remarks \\
\hline $\begin{array}{l}\text { Huang et al. } \\
\text { [69] and Koes- } \\
\text { dwiady et al. } \\
\text { [17] }\end{array}$ & $\begin{array}{l}\text { Transport } \\
\text { flow }\end{array}$ & $\begin{array}{l}\text { Real time traffic data in California (PeMS } \\
\text { dataset) and entrance-exit station data of a } \\
\text { highway }\end{array}$ & DBN & $\begin{array}{l}\text { Traffic flow prediction; [69] incorporated } \\
\text { multitask learning in DBN; [17] combined } \\
\text { weather parameters to improve prediction } \\
\text { performance. }\end{array}$ \\
\hline $\begin{array}{l}\text { Lv et al. [5] } \\
\text { and Yang et al. } \\
\text { [93] }\end{array}$ & $\begin{array}{l}\text { Transport } \\
\text { flow }\end{array}$ & $\begin{array}{l}\text { Real time traffic data in California (PeMS } \\
\text { dataset) }\end{array}$ & SAE & $\begin{array}{l}\text { Traffic flow prediction; [5] used stacked } \\
\text { autoencoders to learn traffic features for } \\
\text { prediction; [93] used Levenberg-Marquardt } \\
\text { Algorithm to train the deep learning model. }\end{array}$ \\
\hline $\begin{array}{l}\text { Tian et al.[40] } \\
\text { and Ma et al. } \\
\text { [41] }\end{array}$ & $\begin{array}{l}\text { Transport } \\
\text { flow }\end{array}$ & $\begin{array}{l}\text { Real time traffic data in California (PeMS } \\
\text { dataset) and } 42387 \text { records of travel speed } \\
\text { data in Beijing }\end{array}$ & RNN & $\begin{array}{l}\text { Traffic flow/speed prediction; used LSTM } \\
\text { to model long-term dependencies. }\end{array}$ \\
\hline Song et al. [68] & $\begin{array}{l}\text { Human } \\
\text { mobility }\end{array}$ & $\begin{array}{l}\text { 1.6 million GPS records in Japan and trans- } \\
\text { portation network data (i.e. road structure } \\
\text { and POI information) }\end{array}$ & RNN & $\begin{array}{l}\text { City-wide human mobility prediction; used } \\
\text { multi-task LSTM to jointly learn people } \\
\text { movement and transportation mode pat- } \\
\text { terns. }\end{array}$ \\
\hline Zhang et al. [6] & $\begin{array}{l}\text { Human } \\
\text { mobility }\end{array}$ & $\begin{array}{l}\text { GPS trajectories data, bike trip data, } \\
\text { weather conditions and events }\end{array}$ & CNN & $\begin{array}{l}\text { Forecasts the inflow and outflow of crowds } \\
\text { in each region of a city; used ResNet to } \\
\text { model spatial correlation; collected times- } \\
\text { tamp data to model temporal correlation. }\end{array}$ \\
\hline Xu et al. [120] & $\begin{array}{l}\text { Human } \\
\text { mobility }\end{array}$ & $\begin{array}{l}600 \text { millions taxi trip data contains GPS lo- } \\
\text { cation and timestamp of pick-up and drop- } \\
\text { off event in NYC }\end{array}$ & RNN & $\begin{array}{l}\text { Taxi demand prediction; predicted future } \\
\text { taxi demand in each area of a city based } \\
\text { on the recent demand and other relevant } \\
\text { information, e.g., weather, time, and drop- } \\
\text { offs. }\end{array}$ \\
\hline $\begin{array}{l}\text { Wang et al. } \\
\text { [44] }\end{array}$ & $\begin{array}{l}\text { Human } \\
\text { mobility }\end{array}$ & $\begin{array}{l}2 \text { billion GPS trajectories with time, driver } \\
\text { ID and weather condition }\end{array}$ & Hybrid & $\begin{array}{l}\text { Travel time estimation; introduced a CNN- } \\
\text { RNN hybrid approach to model both spa- } \\
\text { tial and temporal information. }\end{array}$ \\
\hline $\begin{array}{l}\text { Amato } \quad \text { et } \\
\text { al. [87] and } \\
\text { Valipour et al. } \\
\text { [71] }\end{array}$ & Parking & $\begin{array}{l}\text { CNRPark and PKLot datasets contain im- } \\
\text { ages of parking lots and segmented parking } \\
\text { spaces }\end{array}$ & CNN & $\begin{array}{l}\text { Parking lot occupancy detection; applied } \\
\text { AlexNet and VGGNet, respectively. }\end{array}$ \\
\hline
\end{tabular}

TABLE 2

Overview of research using deep learning for transportation related applications.

outflow of crowds in each region of a city based on data from different sources, such as GPS trajectories, bike trips, weather conditions and events.

Predicting taxi demand can help better organise taxi fleets and minimise wait-time for passengers and drivers. The work in [120] proposed a LSTM based RNN model for taxi demand prediction in each areas of a city, based on the data collected from around 600 millions taxi trips during 2013 and 2016 at the New York City. Future taxi demand was predicted based on not only the recent demand but also other relevant information, e.g., weather, time and pick-up and drop-off events. GPS trajectories also can be used to estimate travel time in a city. The work in [44] proposed a hybrid deep learning framework $(\mathrm{CNN}+\mathrm{RNN})$ to estimate travel time on any route in a city. CNN was used to capture the spatial dependency of taxi trajectories and RNN was used to model the temporal dependencies among these trajectories. It collected about 2 billion GPS trajectories together with the corresponding time information and driver IDs in Beijing and Chengdu. The travel time of both the entire path or each local path was then estimated based on the GPS trajectories, weather, time and driver information using the hybrid model.

\subsubsection{Parking}

As the number of vehicles in cities keeps increasing, finding a parking space efficiently has become a problem for drivers. The idea of smart parking has been introduced to optimise parking space usage, improve the efficiency of parking operations, alleviate traffic congestion, and reduce $\mathrm{CO}$ emissions. Vacant parking space detection using only visual informa- tion is still an open problem. Many conventional techniques are only tailored and fine-tuned to specific and ideal contexts and scenarios [122], [108]; therefore, they cannot be easily generalised, or be adapted to a different parking lot. In contrast, with deep learning models, especially $\mathrm{CNN}$, the result is robust to disturbance created by partial occlusions, presence of shadows and variation of light conditions.

The work in [87] proposed a decentralised and efficient solution for visual parking lot occupancy detection based on an AlexNet. The system was deployed at the parking lot of the research campus in Pisa as part of the smart city application. Similarly, the work in [71] presented a robust parking lot detection algorithm based on a light version of VGGNet. It was used to report the occupancy status of a parking stall based on the images taken by cameras. A fully functional system including server-side image analysis and frontend user interface was developed as a smart parking application.

\subsection{Healthcare}

In recent years, many smart healthcare applications have been developed to improve traditional healthcare systems and provide better support for prevention, diagnosis, and treatment of disease. Due to the large number of diseases presented in the existing research we categorised the review based on the nature of medical data: medical sensing, medical informatics, and medical imaging.

\subsubsection{Medical Sensing}

Medical sensing data collected from pervasive sensors, such as smartphone, wearable, and ambient sensors, has 


\begin{tabular}{|c|c|c|c|c|}
\hline Reference & Application & Data & Method & Remarks \\
\hline $\begin{array}{l}\text { Pouladzadeh } \\
\text { et al. [123] }\end{array}$ & $\begin{array}{l}\text { Medical sens- } \\
\text { ing }\end{array}$ & 10000 high-resolution food images & CNN & Food calorie estimation. \\
\hline $\begin{array}{l}\text { Huang et al. } \\
\text { [124] }\end{array}$ & $\begin{array}{l}\text { Medical sens- } \\
\text { ing }\end{array}$ & $\begin{array}{l}\text { UCF101 and HMDB-51 datasets about el- } \\
\text { derly and children care }\end{array}$ & CNN & $\begin{array}{l}\text { Recognised human actions such as falling } \\
\text { to floor and baby crawling; a three stream } \\
\text { convolution neural network was proposed. }\end{array}$ \\
\hline $\begin{array}{l}\text { molchanov et } \\
\text { al. [96] }\end{array}$ & $\begin{array}{l}\text { Medical sens- } \\
\text { ing }\end{array}$ & $\begin{array}{l}\text { multimodal dynamic hand gesture dataset } \\
\text { captured with depth, color and stereo-IR } \\
\text { sensors }\end{array}$ & Hybrid & $\begin{array}{l}\text { Hand gestures detection and classification; } \\
\text { online system without noticeable lag; a re- } \\
\text { current 3D CNN was proposed. }\end{array}$ \\
\hline $\begin{array}{l}\text { Hammerla et } \\
\text { al. [125] }\end{array}$ & $\begin{array}{l}\text { Medical sens- } \\
\text { ing }\end{array}$ & $\begin{array}{l}\text { Dataset collected from } 34 \text { people with } \\
\text { parkinson disease }\end{array}$ & DBN & Parkinson disease state assessment. \\
\hline $\begin{array}{l}\text { Wulsin et al. } \\
\text { [88] and Ren et } \\
\text { al. [90] }\end{array}$ & $\begin{array}{l}\text { Medical sens- } \\
\text { ing }\end{array}$ & $\begin{array}{l}\text { EEG data sampled at } 256 \mathrm{~Hz} \text { from } 11 \text { pa- } \\
\text { tients ; three datasets from previous BCI } \\
\text { competition }\end{array}$ & DBN & $\begin{array}{l}\text { EEG data classification and anomaly de- } \\
\text { tection; [90] applied convolutional DBN to } \\
\text { learn features from high-dimensional and } \\
\text { multichannel EEG signals. }\end{array}$ \\
\hline $\begin{array}{l}\text { Acharya et al. } \\
\text { [126] }\end{array}$ & $\begin{array}{l}\text { Medical sens- } \\
\text { ing }\end{array}$ & $\begin{array}{l}\text { Physikalisch-Technische Bundesanstalt di- } \\
\text { agnostic ECG database }\end{array}$ & CNN & $\begin{array}{l}\text { Automated detection of myocardial infarc- } \\
\text { tion from ECG beats (with noise and with- } \\
\text { out noise). }\end{array}$ \\
\hline Lipton et al. [8] & $\begin{array}{l}\text { Medical infor- } \\
\text { matics }\end{array}$ & $\begin{array}{l}\text { Anonymised clinical time series extracted } \\
\text { from the EHR system at Children's Hospital } \\
\text { LA }\end{array}$ & RNN & $\begin{array}{l}\text { Multilabel classification of diagnoses; in- } \\
\text { cluded drop out, target replication and aux- } \\
\text { iliary output. }\end{array}$ \\
\hline $\begin{array}{l}\text { Miotto et al. } \\
\text { [9] }\end{array}$ & $\begin{array}{l}\text { Medical infor- } \\
\text { matics }\end{array}$ & $\begin{array}{l}\text { Aggregated EHRs of about } 700,000 \text { patients } \\
\text { from the Mount Sinai data warehouse }\end{array}$ & SAE & $\begin{array}{l}\text { Assessing the probability of patients to de- } \\
\text { velop various diseases; used stacked de- } \\
\text { noising autoencoder to capture hierarchical } \\
\text { regularities and dependencies. }\end{array}$ \\
\hline Suk et al. [105] & $\begin{array}{l}\text { Medical imag- } \\
\text { ing }\end{array}$ & $\begin{array}{l}\text { Different types of neuroimaging modality } \\
\text { data from ADNI dataset }\end{array}$ & SAE & $\begin{array}{l}\text { Brain disease diagnosis; SAE for latent fea- } \\
\text { ture extraction on a large set of hand-crafted } \\
\text { features. }\end{array}$ \\
\hline Hoo et al. [99] & $\begin{array}{l}\text { Medical imag- } \\
\text { ing }\end{array}$ & $\begin{array}{l}\text { Public available thoracoabdominal lymph } \\
\text { node datasets and interstitial lung disease } \\
\text { dataset }\end{array}$ & CNN & $\begin{array}{l}\text { Anomaly detection; Compared architec- } \\
\text { tures for detecting interstitial disease and } \\
\text { lymph nodes. }\end{array}$ \\
\hline $\begin{array}{l}\text { Ronneberger } \\
\text { et al. [100] }\end{array}$ & $\begin{array}{l}\text { Medical imag- } \\
\text { ing }\end{array}$ & $\begin{array}{l}\text { Datasets from EM segmentation challenge } \\
\text { and ISBI cell tracking challenge }\end{array}$ & CNN & $\begin{array}{l}\text { Cell segmentation; U-Net with deformation } \\
\text { augmentation. }\end{array}$ \\
\hline $\begin{array}{l}\text { Zhou et al. } \\
\text { [101] }\end{array}$ & $\begin{array}{l}\text { Medical imag- } \\
\text { ing }\end{array}$ & $\begin{array}{l}\text { C3H10T1/2 and C2C12 Datasets from the } \\
\text { CMU cell image analysis group }\end{array}$ & CNN & $\begin{array}{l}\text { Cell mitosis detection; 3D convolution } \\
\text { kernel for processing consecutive image } \\
\text { frames. }\end{array}$ \\
\hline
\end{tabular}

TABLE 3

Overview of research using deep learning for healthcare related applications.

been used to develop many different types of applications, ranging from health condition assessment, infant/elderly/patient care to disease diagnosis. As accurate estimation of food intake and daily energy consumption can help solve obesity and improve personal health, the work in [123] proposed a smartphone-based, assistive system to help patients and doctors control diet-related health conditions. The system allowed users to take images of food and then used CNN to classify the food images and automatically calculate the amount of calorie intake.

Healthcare of infant, elderly and people with disabilities or chronic diseases can be improved with medical sensing data and human activity recognition techniques. In [124], an intelligent surveillance system was developed to recognise human activities such as falling to floor and baby crawling. A three stream CNN was proposed for activity recognition, in which the spatial stream CNN was trained for human detection; a temporal stream CNN was trained for activity recognition; and a moving stream net was used to detect speed and direction of the movement. Recognition of hand gestures (e.g., sign language interpretation) is important for people with hearing disabilities. In [96], a recurrent $3 \mathrm{~d}$ convolutional neural network was proposed to perform simultaneous detection and classification of dynamic hand gestures. Some diseases are related to the change of certain body movements such as Parkinson disease; and wearable sensors can be used to detect these movements. In [125], an assessment system based on DBN is developed to differen- tiate Parkinson disease states with data collected from 34 people with Parkinson disease in naturalistic settings.

For critically ill patients, continuous monitoring of vital signs, e.g., electroencephalography (EEG), Electrocardiography (ECG), blood pressure, respiratory rate and body temperature, is extremely important to improve treatment outcomes. EEG has been used to record electrical activities of the brain, and has played important roles in diagnosing epilepsy, sleep disorders, coma and brain death. The research in [88] proposed a DBN based method to detect and visualise anomalies from large EEG datasets. As features of anomalies in training data are usually poorly learned, the authors applied RBM and DBN to detect abnormal EEG waveforms that have high reconstruction errors. Considering the high-dimensional and multichannel nature of EEG data, some studies also applied convolutional DBN [90] and variational autoencoder [127] to learn features from the unlabelled EEG data for EEG signal classification. ECG, which records electrical activities of hearts, has been used to diagnose various cardiovascular diseases. The work in [126] implemented a convolutional neural network for automated detection of myocardial infarction from ECG beats with noise and without noise.

\subsubsection{Medical Informatics}

Medical Informatics helps enhance healthcare quality by analysing large amount of aggregated and cumulated medical data (mainly textual). Electronic health record (EHR) is a 
type of such data containing well-structured, textual information about patients, such as diagnosis, medical history, medications and allergies, immunisation records, vital signs and laboratory test results. Efficient mining of EHR can provide valuable insight into disease management. The work in Lipton et al. [8] applied RNN with LSTM units to classify diagnosis (formulated as a multi-label classification task) based on Pediatric Intensive Care Unit (PICU) time series data (e.g., body temperature, heart rate, blood pressure, and others). Miotto et al. [9] developed a system called "Deep Patient" to automatically learn features from a large-scale EHR database without additional human efforts. A stacked de-noising autoencoder architecture was adopted to capture hierarchical regularities and dependencies. The system was able to accurately predict the probability of a patient in developing a specific disease, e.g., diabetes, schizophrenia and cancer.

\subsubsection{Medical Imaging}

Medical imaging is an important technique in creating visual representations of the interior of human bodies to facilitate doctors in disease diagnosis and treatment. Automated image analysis has become mandatory to modern healthcare because of the amount of imaging data. Research in this area heavily relies on techniques developed for image processing. It is not surprising to see that deep learning, especially $\mathrm{CNN}$ based methods, have been adopted rapidly due to their superior performance in common tasks, e.g., disorder classification [105], CT abnormality detection [99], cell segmentation [100], cell mitosis detection [101], foot ulcer classification [128], interstitial lung disease classification [129], and left ventricle segmentation [130]. Many other medical imaging tasks can also be found in applications for detecting and diagnosing neuronal, ophthalmic, pulmonary, and cardiac diseases. We refer the readers to [34] which provides a comprehensive overview of deep learning techniques for medical imaging from both an application perspective and methodology driven perspective.

\subsection{Environment}

Rapid global urbanisation has posed great threats (e.g., air pollution, extreme weather, wild fire and earthquake) to the environment that we live in. Effective monitoring and protection of environment has been one of most significant tasks for smart cities. Through continuous analysis of the environment related data, e.g., meteorological sensing data, remote sensing images and even social media data, we can have accurate monitoring and forecasting, and identify causes for the problems; more importantly, issue prewarning, better organise resources for rescue and avoid catastrophic effects in natural disasters. We focus on the research related to air quality and disaster (man-made and natural).

\subsubsection{Air Quality}

It is reported by the National Oceanic and Atmospheric Administration (NOAA) that the U.S. spends tens of billions of dollars each year to reduce air pollution in order to protect public health and environment [131]. However, poor air quality is still the main cause for cardiovascular and respiratory diseases which results in tens of thousands deaths across the U.S. annually. Accurate prediction of air quality is a challenging task as it is affected by multiple factors such as meteorology, traffic, location, time and social events. Taking into consideration different factors, the work in [11] proposed a DNN (deep neural network with fusion components) based approach to predict the Air Quality Index (AQI), which is a widely used metric to indicate how polluted the air is. A spatial transformation component is used to address spatial correlation and a distributed fusion network is used to merge all the influential factors. The work in [10] aimed to predict PM2.5 concentration for 52 cities in Japan. It used the historical PM 2.5 concentrations along with other features (e.g., wind speed and rain precipitations) to compute the PM2.5 concentration levels several hours ahead. In their work, the deep model is pre-trained with an AE specifically designed for time series data, and fine-tuned using RNN. The results showed that the method outperformed VENUS, the office PM2.5 prediction system developed by the National Institute for Environmental Studies in Japan. The study in [12] presented a system called Deep Air Learning based SAE to unify air quality feature selection, prediction and interpolation. An sparse layer on the top of the input layer was introduced to perform feature selection, and spatio-temporal information was embedded into the output layer. Experiments on real world data showed the model provided promising prediction results in each regions of Beijing when a haze happened in December 2015.

\subsubsection{Disaster}

Images collected from remote sensing devices and surveillance cameras are important data sources for disaster analysis. The work in [106] aimed to recognise landslide from optical remote sensing images. It applied a stacked autoencoder and wavelet transformation to extract hidden and distinct features and a softmax classifier for prediction. In [47] the authors designed a multiple kernel learning framework based on features extracted from CNN and 3D point clouds. The method was used to detect severe building damages caused by destructive disasters (e.g., earthquakes) from oblique aerial images taken by manned aircrafts and UAVs. The work in [70] proposed an early fire detection system that can detect fire in both indoor and outdoor environments. It used a pre-trained Alexnet for real time fire detection based on images captured from surveillance cameras.

Social media data can complement data collected from physical devices in many aspects. In times of crisis, people may use social media platforms to publish situational updates, find useful information, and seek for help. Efficient and accurate analysis can help city authorities gain situational awareness of the affected citizens, and identify critical infrastructure damage and medical emergencies. The work in [56] presented such an application to identify disaster events from relevant tweets during crisis. A CNN based framework was adopted to capture the salient $n$-gram information by convolution and pooling operations. Results showed that the deep learning based method substantially outperformed the conventional supervised learning methods (e.g., Support Vector Machines, Random Forest, and 


\begin{tabular}{|c|c|c|c|c|}
\hline Reference & Application & Data & Method & Remarks \\
\hline Yi et al. [11] & Air quality & $\begin{array}{l}\text { Hourly air pollutants, meteorological data } \\
\text { and weather forecast data in China }\end{array}$ & DNN & $\begin{array}{l}\text { Air quality prediction; Hand-crafted spa- } \\
\text { tial transformation component to address } \\
\text { spatial correlation; fusion network to fuse } \\
\text { different factors. }\end{array}$ \\
\hline Ong et al. [10] & Air quality & $\begin{array}{l}\text { PM2.5 prediction system called VENUS de- } \\
\text { veloped by the National Institute for Envi- } \\
\text { ronmental Studies in Japan }\end{array}$ & Hybrid & $\begin{array}{l}\text { PM2.5 concentration prediction; pre- } \\
\text { training with auto-encoder; use RNN to } \\
\text { model time series. }\end{array}$ \\
\hline Qi et al. [12] & Air quality & $\begin{array}{l}\text { Hourly meteorological data (e.g. tempera- } \\
\text { ture, humidity, and etc.) and air quality data } \\
\text { (e.g. PM2.5, PM10, and etc.) in Beijing }\end{array}$ & SAE & $\begin{array}{l}\text { Air quality prediction; a SAE model for air } \\
\text { quality feature selection, interpolation and } \\
\text { prediction. }\end{array}$ \\
\hline Liu et al. [106] & Disaster & $\begin{array}{l}\text { An optical remote sensing image set with } \\
1200 \text { samples from Google Earth }\end{array}$ & SAE & $\begin{array}{l}\text { Landslide recognition; a stacked denosing } \\
\text { autoencoder and discrete wavelet transfor- } \\
\text { mation were used to extract features. }\end{array}$ \\
\hline $\begin{array}{l}\text { Vetrivel et al. } \\
\text { [47] }\end{array}$ & Disaster & $\begin{array}{l}\text { Two groups of datasets based on multi-view } \\
\text { oblique images from manned aircrafts and } \\
\text { UAVs }\end{array}$ & CNN & $\begin{array}{l}\text { Disaster damage detection; a multiple- } \\
\text { kernel-learning framework combines CNN } \\
\text { features and 3D point cloud features. }\end{array}$ \\
\hline $\begin{array}{l}\text { Muhammad et } \\
\text { al. [70] }\end{array}$ & Disaster & $\begin{array}{l}\text { A dataset of } 68457 \text { images collected from } \\
\text { different fire datasets of both images and } \\
\text { videos }\end{array}$ & $\mathrm{CNN}$ & $\begin{array}{l}\text { Fire detection in indoor and outdoor en- } \\
\text { vironments; a prioritisation mechanism to } \\
\text { change the priority of camera nodes; dy- } \\
\text { namic channel selection algorithm. }\end{array}$ \\
\hline $\begin{array}{l}\text { Nguyen et al. } \\
\text { [56] }\end{array}$ & Disaster & $\begin{array}{l}\text { Labelled twitter datasets: CrisisNLP, Cri- } \\
\text { sisLex, and AIDR }\end{array}$ & CNN & $\begin{array}{l}\text { Crisis-Related tweets classification with } \\
\text { pre-trained word embeddings. }\end{array}$ \\
\hline
\end{tabular}

Overview of research using deep learning for environment related applications.

Logistic Regression) with current and previous event (outof-event data) data.

\subsection{Public safety}

Smart city also aims to provide safer environments for citizens. With the installation of numerous surveillance cameras and the generated image and video data, enforcement of relevant regulations, criminal investigation and transportation safety can be significantly improved. Research in this aspect heavily replies on techniques developed for image/video processing, e.g., object recognition and identification. This is also one of the reasons that CNN based framework has been extensively used as illustrated in Table 5. We focus on the two important applications of face recognition and traffic surveillance.

\subsubsection{Face Recognition}

Human face conveying identity of a person plays an important role in social interactions. Automated face recognition techniques have been developed for a variety of applications requiring efficient and "smart" interactions with different environments such as rooms, cars, and office desks [132]. It is the process of uniquely verifying (1:1 match) or identifying (1:N match) a person by comparing and analysing patterns based on the person's facial contours.

Many conventional methods for face verification represent faces with over-complete low-level features and employ shallow models, for example, in [133], each face image was encoded into 26K learning-based (LE) descriptors, and then the L2 distance between the LE descriptors was calculated after performing Principle Component Analysis (PCA). Chen et al. [110] extracted 100K LBP descriptors at dense facial landmarks with multiple scales and used Joint Bayesian for verification after PCA. Recent research showed that $\mathrm{CNN}$ has been effective in automatically extracting high-level visual features and significantly outperformed conventional methods in face recognition.
To achieve ultimate accuracy, most of the CNN models tend to be deeper or multiple local facial patch ensemble, which result in prolonged training time and waste of storage space. To alleviate the problem, Sun et al. [102] proposed a light CNN framework to learn a compact embedding for face representation. Features extracted from different face regions were complementary and further boosted the performance. It achieved $97.45 \%$ face verification accuracy on Labeled Faces in the Wild (LFW) dataset, while only requiring weakly aligned faces. In [103] Schroff et al. presented a unified system called FaceNet for face verification (is this the same person), recognition (who is this person) and clustering (find common people among these faces). The method was based on learning a Euclidean embedding per image using CNN, which was trained such that the squared L2 distances in the embedding space directly correspond to face similarity: faces of the same person have small distances and faces of different people have large distances. CNN have also been applied to face age estimation system for access control and surveillance monitoring tasks, e.g., preventing children from entering unauthorised or dangerous zones, preventing teenagers under legal age from buying cigarette from vending machines [134].

\subsubsection{Traffic surveillance}

Traffic surveillance cameras have been widely deployed in city roads, generating numerous images and videos for vehicle detection, tracking, classification and verification [135], which can be used in many applications, such as traffic law enforcement, electronic payment systems, automatic toll collection and traffic monitoring systems. Many conventional methods are based on detection of the license plate for Region of Interest (ROI) extraction [114], [115], [15]. Unfortunately, in many occasions license plate of a vehicle may not be discovered because the plate might be missing, cloned or simply unrecognisable.

Automatic vehicle detection and recognition aims to process videos recorded from stationary cameras over roads 


\begin{tabular}{|c|c|c|c|c|}
\hline Reference & Application & Data & Method & Remarks \\
\hline Sun et al. [102] & $\begin{array}{l}\text { Face recogni- } \\
\text { tion }\end{array}$ & Labeled Faces in the Wild (LFW) & CNN & $\begin{array}{l}\text { Face verification; a set of high-level feature } \\
\text { representations learned through deep learn- } \\
\text { ing, referred to as Deep hidden IDentity } \\
\text { features (DeepID). }\end{array}$ \\
\hline $\begin{array}{l}\text { Schroff et al. } \\
\text { [103] }\end{array}$ & $\begin{array}{l}\text { Face recogni- } \\
\text { tion }\end{array}$ & $\begin{array}{l}\text { Labeled Faces in the Wild (LFW) and } \\
\text { YouTube Faces DB }\end{array}$ & $\mathrm{CNN}$ & $\begin{array}{l}\text { Face verification, recognition and cluster- } \\
\text { ing; using CNN to directly optimise the em- } \\
\text { bedding, rather than an intermediate bottle- } \\
\text { neck layer as in conventional CNNs. }\end{array}$ \\
\hline $\begin{array}{l}\text { Menotti et al. } \\
\text { [83] }\end{array}$ & $\begin{array}{l}\text { Traffic surveil- } \\
\text { lance }\end{array}$ & $\begin{array}{l}\text { The dataset was obtained from Brazilian li- } \\
\text { cense plate images captured in a real-world } \\
\text { setting }\end{array}$ & $\mathrm{CNN}$ & $\begin{array}{l}\text { Vehicle license plate recognition; Using two } \\
\text { networks, one for recognition of digits and } \\
\text { the other for letters; was the first time that a } \\
\text { random CNN was used for vehicle identifi- } \\
\text { cation. }\end{array}$ \\
\hline $\begin{array}{l}\text { Shivakumara } \\
\text { et al. [97] }\end{array}$ & $\begin{array}{l}\text { Traffic surveil- } \\
\text { lance }\end{array}$ & $\begin{array}{l}20105 \text { images for experimentation in this } \\
\text { work, } 18270 \text { images from UCSD dataset, } \\
1835 \text { images from MIMOS dataset }\end{array}$ & Hybrid & $\begin{array}{l}\text { Vehicle license plate recognition; combined } \\
\text { merits of both CNN and RNN to handle } \\
\text { issues of poor quality, complex background, } \\
\text { blur and noise. }\end{array}$ \\
\hline $\begin{array}{l}\text { Sochor et al. } \\
\text { [104] }\end{array}$ & $\begin{array}{l}\text { Traffic surveil- } \\
\text { lance }\end{array}$ & $\begin{array}{l}\text { Boxcars dataset with the 3D bounding } \\
\text { boxes and contains } 21250 \text { vehicles ( } 63750 \\
\text { images) of } 27 \text { different makes. }\end{array}$ & CNN & $\begin{array}{l}\text { Vehicle detection and recognition; collected } \\
\text { and annotated a new dataset BoxCars; } \\
\text { showed that additional information easily } \\
\text { obtainable in real time from static surveil- } \\
\text { lance cameras can boost the CNN verifica- } \\
\text { tion performance greatly. }\end{array}$ \\
\hline Liu et al. [13] & $\begin{array}{l}\text { Traffic surveil- } \\
\text { lance }\end{array}$ & $\begin{array}{l}\text { VeRi-776 dataset contains about } 40000 \text { im- } \\
\text { ages of } 619 \text { vehicles captured by } 20 \text { surveil- } \\
\text { lance cameras. }\end{array}$ & $\mathrm{CNN}$ & $\begin{array}{l}\text { Vehicle re-identification; collected the } \\
\text { dataset VeRi-776; proposed a deep learning- } \\
\text { based, progressive vehicle re-identification } \\
\text { approach for urban surveillance. }\end{array}$ \\
\hline
\end{tabular}

Overview of research using deep learning for public safety related applications.

(e.g. CCTV cameras installed nearby traffic intersections and junctions) and then transmit them to the surveillance centre for recording and processing. Sochor et al. [104] presented an automatic vehicle detection and recognition method which saves a lot of time and effort for users trying to identify blacklisted vehicles from a large surveillance image database. This work showed that additional information easily obtainable in real time from static surveillance cameras can boost the $\mathrm{CNN}$ verification performance greatly. Recently, the application of vehicle re-identification (re-ID) has gained considerable research attention. Liu et al. [13] proposed a vehicle re-ID system to quickly discover, locate and track the target vehicles across multiple cameras. It employed CNN to extract appearance attributes as a coarse filter, and Siamese neural network to accurately verify vehicles based on the license plate. The work reported $9.28 \%$ improvement over the state-of-the-art methods in terms of mean average precision.

\section{Challenges, Future Research and Con- CLUDING REMARKS}

In what follows we highlight several challenging issues and future research directions based on our investigation, and then conclude the paper.

\subsection{Efficient Deep Learning Models}

Research on deep leaning from smart city data is still in an early stage. Researchers have applied different basic deep learning architectures in solving many practical problems and achieved promising results. The primary concern in these studies has been high accuracy (e.g., for classification) or low error (e.g., for prediction). Computation complexity, e.g., training cost, has been largely ignored. It also seems that the researchers have not been fully aware of some latest development in deep learning. As network architectures become deeper and deeper, learning tends to be extremely hard and inefficient. Much of the recent research emphasises on learning efficiency, for example, Deep Residual Network (ResNet) in [27] introduced shortcut connections that allow convolution neural networks to have super deep structures without significantly increasing computation complexity. Transformer based methods [136] used only attention mechanisms with position embeddings to learn representations from sequence data. It not only improved the state-of-theart in the area of machine translation but also achieved signification improvement in training time. Likewise, we hope that smart city researchers pay attention to the learning efficiency in designing deep models and include computation complexity as the additional evaluation metric.

\subsection{Emerging Deep Learning Paradigms}

As indicated in [21], unsupervised learning will become far more important in the longer term, as human and animal learning is largely unsupervised and they discover the structure of the world mainly by observation. This is particular the case for smart city data, which is all about observation and most of which is unlabelled. Generative adversarial network [137] is a promising unsupervised paradigm originally designed in the field of image processing. It is an effective (unsupervised) generative framework that has two competing neural networks (i.e., generator and discriminator) trained simultaneously. It has been used to generate photographs that look at least superficially authentic to human observers. It also has great potential in smart city applications to address issues, such as missing sensor data estimation, anomaly detection for environment and 
transportation, vehicle identification and re-identification in public safety, and many others.

Transfer learning is a learning architecture that gained increasing attentions recently. In practical applications, very often the labelled datasets may be too specific or small to train effective models from scratch. In this case transfer learning can be applied: first learning an approximate model from a pre-trained network on a large related dataset and then fine-tuning the network with the data for the specific task. For example, knowledge gained by learning to recognise many classes with a large dataset could apply to recognise specific classes. In a sense, transfer learning encourages reuse of existing learned models and has the potential to save considerable computation cost. Data in smart city is highly distributed; for one application domain, data collected from different geographical locations shares much commonality. This characteristic makes transfer learning particularly attractive for more efficient data analytics. In fact, it has been applied in a limited number of small-scale studies such as fire detection [70] and parking lot detection [71]. In the future, we would expect extensive applications of transfer learning in many other smart city domains.

Developing effective deep models requires substantial efforts in architecture engineering which is time consuming and expensive process. Neural Architecture Search (NAS) is an emerging deep learning paradigm that aims to automatically find or design effective deep architectures for specific tasks minimising human participation. The interesting research direction has quickly drawn considerable attention of researchers from computational intelligence, in particular, evolutionary computing. Recently, evolutionary algorithms (e.g., genetic programming) [138], [139], [140], [141] and reinforcement learning based methods [142], [143] have been used to design new neural architectures. Research has reported that the deep models found by NAS can outperform a large number of manually designed models. Although the current NAS methods mostly focus on image classification tasks, we believe that the paradigm will be applied to other deep models and used for the smart city domains in the near future.

\subsection{Privacy in Smart Cities}

While smart city applications promise to make our living environment safer, greener and more sustainable, they also bring challenges regarding citizens' privacy. With so much data collected, stored, analysed and used, often by unknown parties, citizens seriously worry about their personal information being misused or abused by governments and companies. Rather than awaiting for the laws and regulations to be made, the research community has already started devising various techniques in protecting citizens' private data, e.g., data distribution, data modification, data mining algorithm, data or rule hiding, privacy preservation [144]. Recently, for one of the most sensitive data types, i.e., medical data, studies have applied the Generative Adversarial Network to produce high quality synthetic patient records [145] and location based data [146] to protect personal sensitive information, and reported promising results on real data in many experiments. However, there is no such technique that can preserve citizens' privacy in all situations. On one hand, more advanced techniques for specific applications need to be developed, on the other, laws and regulations for protecting privacy, e.g., the General Data Protection Regulation (GDPR) from EU, are needed.

\subsection{Knowledge Fusion with Deep Learning}

So far, smart city has appeared as a collection of loosely coupled applications in different domains, and little relation can be identified from these applications. The current research is much like applying deep learning to identify and extract superficial knowledge. Data from different domains may be correlated and may affect each other, e.g., bad weather may affect traffic conditions, a traffic congestion may result in extra fuel consumption, air and noise pollution, and such pollution may cause cardiovascular and respiratory disease. However, such correlations have been largely overlooked in the existing research.

Smart city is also a Cyber-Physical-Social system (CPS) and the data from the cyber, physical and social worlds has different characteristics, for example, trustworthiness of the data collected from the social world may be questionable; and data from the physical world usually has low-level semantics and cannot be easily integrated with social data. However, knowledge discovered from different worlds can complement and reinforce each other in order to derive more meaningful insights, e.g., traffic anomaly can be identified by both loop sensors installed on roads and social media data published online. The information from the two different sources varies in nature but provides details complementing each other. We believe that more insightful knowledge can be derived if we can discover the correlations among data from the physical, cyber, and social worlds. This knowledge fusion process requires techniques which can effectively handle cross-domain and multi-modal data. If we were to think a smart city as an integrative system we have to interrelate the outcome of data analytics in each individual domains and fuse the learned knowledge wherever possible to create a system that is truly smart. Deep learning techniques are considered as good candidates [147], [148] for the knowledge fusion.

\subsection{Distributed Intelligence}

The research in [149] proposed the idea of "distributed intelligence", which transfers much of the intelligent computation (e.g., data aggregation, smoothing, reasoning and analytics) from data centres to the much smaller while autonomous units at different levels in a city environment, e.g., sensor nodes, gateways of Wireless Sensor networks, smartphones, or mobile edge networks, in order to tackle the challenges brought by the volume and velocity of big city data. However, we have to take into consideration the capability of these computing devices as they are far less powerful compared to servers in data centres. For deep learning, we envisage the need to develop more lightweight models so they can be implemented on the capabilityconstrained devices. The idea of distributed intelligence also provides great potential for transfer learning, where pretrained models are implanted into distributed devices for fine-tuning. 


\subsection{Limitations of Deep Learning}

Although deep learning has demonstrated great success and outperformed many other conventional machine learning methods, it is not a versatile solution for every applications in smart city. There are at least a number of limitations that smart city researchers have to take into account: 1) deep learning models are notoriously expensive to train, and it is not uncommon for the training to take days even with high-end computing devices. Meanwhile, it is also difficult to configure the models; sometimes many experiments need to be conducted in order to find proper or near optimal configurations; 2) for supervised learning, large amount of training data is needed. In applications where only data of small or medium size is available, performance of a deep learning model may not be guaranteed; and 3) a learned deep model is usually hard to interpret in intuitive ways. It usually works like a "black box" that maps an input to an output, and may not be a good option for applications in which users are interested in knowing how decisions are made.

\subsection{Conclusion}

Smart city data analytics is a complex research topic due to several reasons, such as many different tasks in many different application domains; the tremendous amount of data of different types and modalities, and the spatial and temporal dependencies among the data. We reviewed the existing research on the converging field of deep learning and smart city and aimed to draw a clear landscape for it. From the technique oriented perspective, we presented in detail different basic and extended deep learning models and discussed how these techniques enable knowledge discovery from city data; while from the application oriented perspective, we demonstrated how problems in specific applications can be addressed by various deep learning techniques. We further contributed to the research by identifying a number of challenges and future research directions for this rapidly evolving field, e.g., emerging deep learning paradigms, deep learning based knowledge fusion and privacy preservation. We hope that our study would motivate more smart city researchers to further explore this exciting research topic and develop more creative and computationally practical deep learning models specifically designed for smart city applications.

\section{ACKNOWLEDGMENT}

The authors would like to thank all the anonymous reviewers for their valuable and helpful comments. This research is funded by the Research Development Fund at Xi'an Jiaotong-Liverpool University, contract number RDF-16-0134. It is also partially supported by Jiangsu Planned Projects for Postdoctoral Research Funds under Grant 2018K005A, National Natural Science Foundation of China under Grant 61701418, and Innovation Projects of The Next Generation Internet Technology under Grant NGII20170301.

\section{REFERENCES}

[1] United Nations. World Urbanization Prospects The 2014 Revision Highlights. Technical report, Department of Economic and Social Affairs, United Nations, ST/ESA/SER.A/352, 2014.
[2] Andrea Zanella, Nicola Bui, Angelo Castellani, Lorenzo Vangelista, and Michele Zorzi. Internet of things for smart cities. IEEE Internet of Things Journal, 1(1):22-32, 2014.

[3] Luigi Atzori, Antonio Iera, and Giacomo Morabito. The internet of things: A survey. Computer networks, 54(15):2787-2805, 2010.

[4] Gang Pan, Guande Qi, Wangsheng Zhang, Shijian Li, Zhaohui $\mathrm{Wu}$, and Laurence Tianruo Yang. Trace analysis and mining for smart cities: issues, methods, and applications. IEEE Communications Magazine, 51(6):120-126, 2013.

[5] Yisheng Lv, Yanjie Duan, Wenwen Kang, Zhengxi Li, and FeiYue Wang. Traffic flow prediction with big data: a deep learning approach. IEEE Transactions on Intelligent Transportation Systems, 16(2):865-873, 2015.

[6] Junbo Zhang, Yu Zheng, and Dekang Qi. Deep spatio-temporal residual networks for citywide crowd flows prediction. In $A A A I$, pages 1655-1661, 2017.

[7] Upkar Varshney. Pervasive healthcare and wireless health monitoring. Mobile Networks and Applications, 12(2-3):113-127, 2007.

[8] Zachary C Lipton, David C Kale, Charles Elkan, and Randall Wetzell. Learning to diagnose with LSTM recurrent neural networks. In International Conference on Learning Representations (ICLR 2016), 2015.

[9] Riccardo Miotto, Li Li, Brian A Kidd, and Joel T Dudley. Deep patient: an unsupervised representation to predict the future of patients from the electronic health records. Scientific reports, 6:26094, 2016

[10] Bun Theang Ong, Komei Sugiura, and Koji Zettsu. Dynamically pre-trained deep recurrent neural networks using environmental monitoring data for predicting PM2. 5. Neural Computing and Applications, 27(6):1553-1566, 2016.

[11] Xiuwen Yi, Junbo Zhang, Zhaoyuan Wang, Tianrui Li, and Yu Zheng. Deep distributed fusion network for air quality prediction. In Proceedings of the 24 th SIGKDD conference on Knowledge Discovery and Data Mining, 2018.

[12] Zhongang Qi, Tianchun Wang, Guojie Song, Weisong Hu, Xi Li, and Zhongfei Mark Zhang. Deep air learning: Interpolation, prediction, and feature analysis of fine-grained air quality. IEEE Transactions on Knowledge and Data Engineering, 2018.

[13] Xinchen Liu, Wu Liu, Tao Mei, and Huadong Ma. A deep learning-based approach to progressive vehicle re-identification for urban surveillance. In European Conference on Computer Vision, pages 869-884. Springer, 2016.

[14] Yong Tang, Congzhe Zhang, Renshu Gu, Peng Li, and Bin Yang. Vehicle detection and recognition for intelligent traffic surveillance system. Multimedia tools and applications, 76(4):5817-5832, 2017.

[15] Christos-Nikolaos E Anagnostopoulos, Ioannis E Anagnostopoulos, Ioannis D Psoroulas, Vassili Loumos, and Eleftherios Kayafas. License plate recognition from still images and video sequences: A survey. IEEE Transactions on intelligent transportation systems, 9(3):377-391, 2008.

[16] Rob Kitchin. The real-time city? big data and smart urbanism. GeoJournal, 79(1):1-14, 2014.

[17] Arief Koesdwiady, Ridha Soua, and Fakhreddine Karray. Improving traffic flow prediction with weather information in connected cars: a deep learning approach. IEEE Transactions on Vehicular Technology, 65(12):9508-9517, 2016.

[18] Hongliang Guo, Zhiguang Cao, Madhavan Seshadri, Jie Zhang, Dusit Niyato, and Ulrich Fastenrath. Routing multiple vehicles cooperatively: Minimizing road network breakdown probability. IEEE Transactions on Emerging Topics in Computational Intelligence, 1(2):112-124, 2017.

[19] Kumar Utkarsh, Anupam Trivedi, Dipti Srinivasan, and Thomas Reindl. A consensus-based distributed computational intelligence technique for real-time optimal control in smart distribution grids. IEEE Transactions on Emerging Topics in Computational Intelligence, 1(1):51-60, 2017.

[20] Anuradha Saha, Amit Konar, and Atulya K Nagar. EEG Analysis for Cognitive Failure Detection in Driving Using Type-2 Fuzzy Classifiers. IEEE Transactions on Emerging Topics in Computational Intelligence, 1(6):437-453, 2017.

[21] Yann LeCun, Yoshua Bengio, and Geoffrey Hinton. Deep learning. Nature, 521(7553):436-444, 2015

[22] Ilya Sutskever, Oriol Vinyals, and Quoc V Le. Sequence to sequence learning with neural networks. In Advances in neural information processing systems, pages 3104-3112, 2014. 
[23] Kyunghyun Cho, Bart Van Merrienboer, Caglar Gulcehre, Dzmitry Bahdanau, Fethi Bougares, Holger Schwenk, and Yoshua Bengio. Learning phrase representations using RNN encoder-decoder for statistical machine translation. Empirical Methods in Natural Language Processing, pages 1724-1734, 2014.

[24] Dzmitry Bahdanau, Kyunghyun Cho, and Yoshua Bengio. Neural machine translation by jointly learning to align and translate. In International Conference on Learning Representations (ICLR 2015), 2014.

[25] Alex Krizhevsky, Ilya Sutskever, and Geoffrey E Hinton. Imagenet classification with deep convolutional neural networks. In Advances in neural information processing systems, pages 1097-1105, 2012.

[26] Karen Simonyan and Andrew Zisserman. Very deep convolutional networks for large-scale image recognition. In International Conference on Learning Representations (ICLR 2015), 2014.

[27] Kaiming He, Xiangyu Zhang, Shaoqing Ren, and Jian Sun. Deep residual learning for image recognition. In Proceedings of the IEEE conference on computer vision and pattern recognition, pages 770-778, 2016.

[28] Geoffrey Hinton, Li Deng, Dong Yu, George E Dahl, Abdelrahman Mohamed, Navdeep Jaitly, Andrew Senior, Vincent Vanhoucke, Patrick Nguyen, Tara N Sainath, et al. Deep neural networks for acoustic modeling in speech recognition: The shared views of four research groups. IEEE Signal Processing Magazine, 29(6):82-97, 2012.

[29] Alex Graves, Abdel-rahman Mohamed, and Geoffrey Hinton. Speech recognition with deep recurrent neural networks. In Acoustics, speech and signal processing (icassp), 2013 ieee international conference on, pages 6645-6649. IEEE, 2013.

[30] Dario Amodei, Sundaram Ananthanarayanan, Rishita Anubhai, Jingliang Bai, Eric Battenberg, Carl Case, Jared Casper, Bryan Catanzaro, Qiang Cheng, Guoliang Chen, et al. Deep speech 2: End-to-end speech recognition in english and mandarin. In International Conference on Machine Learning, pages 173-182, 2016.

[31] Yu Zheng, Licia Capra, Ouri Wolfson, and Hai Yang. Urban computing: concepts, methodologies, and applications. $A C M$ Transactions on Intelligent Systems and Technology (TIST), 5(3):38, 2014.

[32] Li Minn Ang and Kah Phooi Seng. Big sensor data applications in urban environments. Big Data Research, 4(C):1-12, 2016.

[33] Li Minn Ang, Kah Phooi Seng, Adamu Zungeru, and Gerald Ijemaru. Big sensor data systems for smart cities. IEEE Internet of Things Journal, PP(99):1-1, 2017.

[34] G Litjens, T Kooi, B. E. Bejnordi, Setio Aaa, F Ciompi, M Ghafoorian, Van Der Laak Jawm, Ginneken B Van, and C. I. Snchez. A survey on deep learning in medical image analysis. Medical Image Analysis, 42(9):60-88, 2017.

[35] Suparna De, Yuchao Zhou, Iker Larizgoitia Abad, and Klaus Moessner. Cyber-physical-social frameworks for urban big data systems: A survey. Applied Sciences, 7(10):1017, 2017.

[36] Michael Compton, Payam Barnaghi, Luis Bermudez, Ral Garca Castro, Oscar Corcho, Simon Cox, John Graybeal, Manfred Hauswirth, Cory Henson, Arthur Herzog, Vincent Huang, Krzysztof Janowicz, W. David Kelsey, Danh Le Phuoc, Laurent Lefort, Myriam Leggieri, Holger Neuhaus, Andriy Nikolov, Kevin Page, Alexandre Passant, Amit Sheth, and Kerry Taylor. The SSN Ontology of the Semantic Sensor Networks Incubator Group. Journal of Web Semantics, 17:2-32, 2012.

[37] Yongrui Qin, Quan Z. Sheng, Nickolas J.G. Falkner, Schahram Dustdar, Hua Wang, and Athanasios V. Vasilakos. When things matter: A survey on data-centric internet of things. Journal of Network and Computer Applications, 64:137 - 153, 2016.

[38] Payam Barnaghi, Wei Wang, Lijun Dong, and Chonggang Wang. A linked-data model for semantic sensor streams. In Green Computing and Communications (GreenCom), 2013 IEEE and Internet of Things (iThings/CPSCom), IEEE International Conference on and IEEE Cyber, Physical and Social Computing, pages 468-475. IEEE, 2013.

[39] Y. Zhou, S. De, W. Wang, and K. Moessner. Enabling query of frequently updated data from mobile sensing sources. In 2014 IEEE 17th International Conference on Computational Science and Engineering, pages 946-952, Dec 2014.

[40] Yongxue Tian and Li Pan. Predicting short-term traffic flow by long short-term memory recurrent neural network. In IEEE International Conference on Smart City/socialcom/sustaincom, pages 153-158, 2015.
[41] Xiaolei Ma, Zhimin Tao, Yinhai Wang, Haiyang Yu, and Yunpeng Wang. Long short-term memory neural network for traffic speed prediction using remote microwave sensor data. Transportation Research Part C: Emerging Technologies, 54:187-197, 2015.

[42] Jindong Wang, Yiqiang Chen, Shuji Hao, Xiaohui Peng, and Lisha Hu. Deep learning for sensor-based activity recognition: A Survey. Pattern Recognition Letters, 2018.

[43] Abdulmajid Murad and Jae-Young Pyun. Deep recurrent neural networks for human activity recognition. Sensors, 17(11):2556, 2017.

[44] Dong Wang, Junbo Zhang, Wei Cao, Jian Li, and Yu Zheng. When Will You Arrive? Estimating Travel Time Based on Deep Neural Networks. In Proceedings of the Thirty-Second AAAI Conference on Artificial Intelligence, 2018.

[45] Naser Hossein Motlagh, Tarik Taleb, and Osama Arouk. Lowaltitude unmanned aerial vehicles-based internet of things services: Comprehensive survey and future perspectives. IEEE Internet of Things Journal, 3(6):899-922, 2016.

[46] Naser Hossein Motlagh, Miloud Bagaa, and Tarik Taleb. UAVbased IoT platform: a crowd surveillance use case. IEEE Communications Magazine, 55(2):128-134, 2017.

[47] Anand Vetrivel, Markus Gerke, Norman Kerle, Francesco Nex, and George Vosselman. Disaster damage detection through synergistic use of deep learning and 3D point cloud features derived from very high resolution oblique aerial images, and multiple-kernel-learning. ISPRS journal of photogrammetry and remote sensing, 140:45-59, 2018.

[48] David G Lowe. Distinctive image features from scale-invariant keypoints. International journal of computer vision, 60(2):91-110, 2004.

[49] Xiaoyu Wang, Tony X Han, and Shuicheng Yan. An HOG-LBP human detector with partial occlusion handling. In Computer Vision, 2009 IEEE 12th International Conference on, pages 32-39. IEEE, 2009.

[50] Amit Sheth. Citizen sensing, social signals, and enriching human experience. IEEE Internet Computing, 13(4), 2009.

[51] Meenakshi Nagarajan, Karthik Gomadam, Amit P Sheth, Ajith Ranabahu, Raghava Mutharaju, and Ashutosh Jadhav. Spatiotemporal-thematic analysis of citizen sensor data: Challenges and experiences. In International Conference on Web Information Systems Engineering, pages 539-553. Springer, 2009.

[52] Eleonora D'Andrea, Pietro Ducange, Beatrice Lazzerini, and Francesco Marcelloni. Real-time detection of traffic from twitter stream analysis. IEEE Transactions on Intelligent Transportation Systems, 16(4):2269-2283, 2015.

[53] Pramod Anantharam, Payam Barnaghi, Krishnaprasad Thirunarayan, and Amit Sheth. Extracting city traffic events from social streams. ACM Transactions on Intelligent Systems and Technology (TIST), 6(4):43, 2015.

[54] Signorini Alessio, Segre Alberto Maria, and Philip M Polgreen. The use of twitter to track levels of disease activity and public concern in the U.S. during the influenza a H1N1 pandemic. Plos One, 6(5):e19467, 2011.

[55] Andrew Crooks, Arie Croitoru, Anthony Stefanidis, and Jacek Radzikowski. Earthquake: Twitter as a distributed sensor system. Transactions in Gis, 17(1):124-147, 2013.

[56] Dat Tien Nguyen, Kamla Al-Mannai, Shafiq R Joty, Hassan Sajjad, Muhammad Imran, and Prasenjit Mitra. Robust classification of crisis-related data on social networks using convolutional neural networks. In ICWSM, pages 632-635, 2017.

[57] Tomas Mikolov, Kai Chen, Greg Corrado, and Jeffrey Dean. Efficient estimation of word representations in vector space. arXiv: Computation and Language, 2013.

[58] Tomas Mikolov, Ilya Sutskever, Kai Chen, Gregory S Corrado, and Jeffrey Dean. Distributed representations of words and phrases and their compositionality. neural information processing systems, pages 3111-3119, 2013.

[59] Jeffrey L Elman. Finding structure in time. Cognitive science, 14(2):179-211, 1990.

[60] Y. Lecun, L. Bottou, Y. Bengio, and P. Haffner. Gradient-based learning applied to document recognition. Proceedings of the IEEE, 86(11):2278-2324, Nov 1998.

[61] Geoffrey E Hinton, Simon Osindero, and Yee-Whye Teh. A fast learning algorithm for deep belief nets. Neural computation, 18(7):1527-1554, 2006.

[62] Yoshua Bengio, Pascal Lamblin, Dan Popovici, and Hugo Larochelle. Greedy layer-wise training of deep networks. In 
Advances in neural information processing systems, pages 153-160, 2007.

[63] Vinod Nair and Geoffrey E Hinton. Rectified linear units improve restricted boltzmann machines. In Proceedings of the 27th international conference on machine learning (ICML-10), pages 807814, 2010.

[64] Herbert Robbins and Sutton Monro. A stochastic approximation method. In Herbert Robbins Selected Papers, pages 102-109. Springer, 1985.

[65] Diederik P. Kingma and Jimmy Ba. Adam: A method for stochastic optimization. In International Conference on Learning Representations (ICLR 2014), 2014.

[66] Tijmen Tieleman and Geoffrey Hinton. Lecture 6.5-rmsprop: Divide the gradient by a running average of its recent magnitude. COURSERA: Neural networks for machine learning, 4(2):26-31, 2012

[67] Ian Goodfellow, Yoshua Bengio, and Aaron Courville. Deep learning, volume 1. MIT press Cambridge, 2016.

[68] Xuan Song, Hiroshi Kanasugi, and Ryosuke Shibasaki. DeepTransport: prediction and simulation of human mobility and transportation mode at a citywide level. In IJCAI, pages 26182624,2016

[69] Wenhao Huang, Guojie Song, Haikun Hong, and Kunqing Xie. Deep architecture for traffic flow prediction: deep belief networks with multitask learning. IEEE Transactions on Intelligent Transportation Systems, 15(5):2191-2201, 2014.

[70] Khan Muhammad, Jamil Ahmad, and Sung Wook Baik. Early fire detection using convolutional neural networks during surveillance for effective disaster management. Neurocomputing, 288:30 42, 2018

[71] Sepehr Valipour, Mennatullah Siam, Eleni Stroulia, and Martin Jagersand. Parking-stall vacancy indicator system, based on deep convolutional neural networks. In Internet of Things, pages 655$660,2016$.

[72] Nitish Srivastava, Geoffrey Hinton, Alex Krizhevsky, Ilya Sutskever, and Ruslan Salakhutdinov. Dropout: a simple way to prevent neural networks from overfitting. The Journal of Machine Learning Research, 15(1):1929-1958, 2014.

[73] Sepp Hochreiter and Jürgen Schmidhuber. Long short-term memory. Neural computation, 9(8):1735-1780, 1997.

[74] Christopher Olah. Understanding LSTM Networks, http:/ / colah.github.io/posts/2015-08-Understanding-LSTMs / . accessed: 2018-09-14.

75] Rui Fu, Zuo Zhang, and Li Li. Using LSTM and GRU neural network methods for traffic flow prediction. In Chinese Association of Automation (YAC), Youth Academic Annual Conference of, pages 324-328. IEEE, 2016.

[76] Jaehyun Ahn, Dongil Shin, Kyuho Kim, and Jihoon Yang. Indoor air quality analysis using deep learning with sensor data. Sensors, 17(11):2476, 2017.

[77] Mike Schuster and Kuldip K Paliwal. Bidirectional recurrent neural networks. IEEE Transactions on Signal Processing, 45(11):26732681, 1997.

[78] Martin Sundermeyer, Tamer Alkhouli, Joern Wuebker, and Hermann Ney. Translation modeling with bidirectional recurrent neural networks. In Proceedings of the 2014 Conference on Empirical Methods in Natural Language Processing (EMNLP), pages 14-25, 2014.

[79] Yong Du, Wei Wang, and Liang Wang. Hierarchical recurrent neural network for skeleton based action recognition. In Proceedings of the IEEE conference on computer vision and pattern recognition, pages 1110-1118, 2015.

[80] Yuxuan Liang, Songyu Ke, Junbo Zhang, Xiuwen Yi, and Yu Zheng. GeoMAN: multi-level attention networks for geosensory time series prediction. In IJCAI, 2018.

[81] Ossama Abdel-Hamid, Abdel-rahman Mohamed, Hui Jiang Li Deng, Gerald Penn, and Dong Yu. Convolutional neural networks for speech recognition. IEEE/ACM Transactions on audio, speech, and language processing, 22(10):1533-1545, 2014.

[82] Jonas Gehring, Michael Auli, David Grangier, Denis Yarats, and Yann N. Dauphin. Convolutional sequence to sequence learning. In ICML, volume 70 of Proceedings of Machine Learning Research, pages 1243-1252. PMLR, 2017.

[83] David Menotti, Giovani Chiachia, Alexandre X Falcão, and VJ Oliveira Neto. Vehicle license plate recognition with random convolutional networks. In Graphics, Patterns and Images (SIBGRAPI), 2014 27th SIBGRAPI Conference on, pages 298-303. IEEE, 2014
[84] Elad Richardson, Matan Sela, Roy Or-El, and Ron Kimmel. Learning detailed face reconstruction from a single image. In Computer Vision and Pattern Recognition (CVPR), 2017 IEEE Conference on, pages 5553-5562. IEEE, 2017.

[85] Xiang Wu, Ran He, and Zhenan Sun. A lightened cnn for deep face representation. In 2015 IEEE Conference on IEEE Computer Vision and Pattern Recognition (CVPR), volume 4, page 5, 2015.

[86] Christian Szegedy, Wei Liu, Yangqing Jia, Pierre Sermanet, Scott Reed, Dragomir Anguelov, Dumitru Erhan, Vincent Vanhoucke, and Andrew Rabinovich. Going deeper with convolutions. In Proceedings of the IEEE conference on computer vision and pattern recognition, pages 1-9, 2015.

[87] Giuseppe Amato, Fabio Carrara, Fabrizio Falchi, Claudio Gennaro, Carlo Meghini, and Claudio Vairo. Deep learning for decentralized parking lot occupancy detection. Expert Systems with Applications, 72, 2016.

[88] Drausin Wulsin, Justin Blanco, Ram Mani, and Brian Litt. Semisupervised anomaly detection for EEG waveforms using deep belief nets. In Machine Learning and Applications (ICMLA), 2010 Ninth International Conference on, pages 436-441. IEEE, 2010.

[89] Honglak Lee, Roger Grosse, Rajesh Ranganath, and Andrew Y. NG. Convolutional deep belief networks for scalable unsupervised learning of hierarchical representations. In Proceedings of the 26th annual international conference on machine learning, pages 609-616. ACM, 2006.

[90] Yuanfang Ren and Yan Wu. Convolutional deep belief networks for feature extraction of EEG signal. In Neural Networks (IJCNN), 2014 International Joint Conference on, pages 2850-2853. IEEE, 2014.

[91] Dumitru Erhan, Yoshua Bengio, Aaron Courville, Pierre-Antoine Manzagol, Pascal Vincent, and Samy Bengio. Why does unsupervised pre-training help deep learning? Journal of Machine Learning Research, 11(Feb):625-660, 2010.

[92] Christopher Poultney, Sumit Chopra, Yann L Cun, et al. Efficient learning of sparse representations with an energy-based model. In Advances in neural information processing systems, pages 11371144, 2007.

[93] Hao-Fan Yang, Tharam S Dillon, and Yi-Ping Phoebe Chen. Optimized structure of the traffic flow forecasting model with a deep learning approach. IEEE transactions on neural networks and learning systems, 28(10):2371-2381, 2017.

[94] Pascal Vincent, Hugo Larochelle, Yoshua Bengio, and PierreAntoine Manzagol. Extracting and composing robust features with denoising autoencoders. In Proceedings of the 25th international conference on Machine learning, pages 1096-1103. ACM, 2008.

[95] Yanjie Duan, Yisheng Lv, Yu-Liang Liu, and Fei-Yue Wang. An efficient realization of deep learning for traffic data imputation. Transportation research part C: emerging technologies, 72:168-181, 2016.

[96] Pavlo Molchanov, Xiaodong Yang, Shalini Gupta, Kihwan Kim, Stephen Tyree, and Jan Kautz. Online detection and classification of dynamic hand gestures with recurrent $3 \mathrm{~d}$ convolutional neural network. In Proceedings of the IEEE Conference on Computer Vision and Pattern Recognition, pages 4207-4215, 2016.

[97] Palaiahnakote Shivakumara, Dongqi Tang, Maryam Asadzadehkaljahi, Tong Lu, Umapada Pal, and Mohammad Hossein Anisi. CNN-RNN based method for license plate recognition. CAAI Transactions on Intelligence Technology, 2018.

[98] Xiaolei Ma, Haiyang Yu, Yunpeng Wang, and Yinhai Wang. Large-scale transportation network congestion evolution prediction using deep learning theory. PloS one, 10(3):e0119044, 2015.

[99] Shin Hoo-Chang, Holger R Roth, Mingchen Gao, Le Lu, Ziyue $\mathrm{Xu}$, Isabella Nogues, Jianhua Yao, Daniel Mollura, and Ronald M Summers. Deep convolutional neural networks for computeraided detection: CNN architectures, dataset characteristics and transfer learning. IEEE transactions on medical imaging, 35(5):1285, 2016.

[100] Olaf Ronneberger, Philipp Fischer, and Thomas Brox. U-net Convolutional networks for biomedical image segmentation. In International Conference on Medical image computing and computerassisted intervention, pages 234-241. Springer, 2015.

[101] Yao Zhou, Hua Mao, and Zhang Yi. Cell mitosis detection using deep neural networks. Knowledge-Based Systems, 137:19-28, 2017.

[102] Yi Sun, Xiaogang Wang, and Xiaoou Tang. Deep learning face representation from predicting 10,000 classes. In Proceedings of the IEEE conference on computer vision and pattern recognition, pages 1891-1898, 2014 
[103] Florian Schroff, Dmitry Kalenichenko, and James Philbin. Facenet: A unified embedding for face recognition and clustering. In Proceedings of the IEEE conference on computer vision and pattern recognition, pages $815-823,2015$.

[104] Jakub Sochor, Adam Herout, and Jiri Havel. Boxcars: 3d boxes as CNN input for improved fine-grained vehicle recognition. In Proceedings of the IEEE Conference on Computer Vision and Pattern Recognition, pages 3006-3015, 2016.

[105] Heung-Il Suk, Seong-Whan Lee, Dinggang Shen, Alzheimers Disease Neuroimaging Initiative, et al. Latent feature representation with stacked auto-encoder for AD/MCI diagnosis. Brain Structure and Function, 220(2):841-859, 2015.

[106] Ying Liu and Linzhi Wu. Geological disaster recognition on optical remote sensing images using deep learning. Procedia Computer Science, 91:566-575, 2016.

[107] SHI Xingjian, Zhourong Chen, Hao Wang, Dit-Yan Yeung, WaiKin Wong, and Wang-chun Woo. Convolutional lstm network: A machine learning approach for precipitation nowcasting. In Advances in neural information processing systems, pages 802-810, 2015.

[108] Qi Wu, Chingchun Huang, Shih-yu Wang, Wei-chen Chiu, and Tsuhan Chen. Robust parking space detection considering interspace correlation. In 2007 IEEE International Conference on Multimedia and Expo, pages 659-662. IEEE, 2007.

[109] Loris Nanni, Alessandra Lumini, and Sheryl Brahnam. Local binary patterns variants as texture descriptors for medical image analysis. Artificial intelligence in medicine, 49(2):117-125, 2010.

[110] Dong Chen, Xudong Cao, Fang Wen, and Jian Sun. Blessing of dimensionality: High-dimensional feature and its efficient compression for face verification. In Proceedings of the IEEE Conference on Computer Vision and Pattern Recognition, pages 3025-3032, 2013.

[111] Kit Yan Chan, Tharam S Dillon, Jaipal Singh, and Elizabeth Chang. Neural-network-based models for short-term traffic flow forecasting using a hybrid exponential smoothing and levenberg-marquardt algorithm. IEEE Transactions on Intelligent Transportation Systems, 13(2):644-654, 2012.

[112] Qeethara Kadhim Al-Shayea. Artificial neural networks in medical diagnosis. International Journal of Computer Science Issues, 8(2):150-154, 2011.

[113] Dimitris Voukantsis, Kostas Karatzas, Jaakko Kukkonen, Teemu Räsänen, Ari Karppinen, and Mikko Kolehmainen. Intercomparison of air quality data using principal component analysis, and forecasting of pm10 and pm2. 5 concentrations using artificial neural networks, in thessaloniki and helsinki. Science of the Total Environment, 409(7):1266-1276, 2011.

[114] Guixiang Liu, Zhongyou Ma, Zhongguo Du, and Can Wen. The calculation method of road travel time based on license plate recognition technology. In Advances in information technology and education, pages 385-389. Springer, 2011.

[115] Yu-Chiun Chiou, Lawrence W Lan, Chun-Ming Tseng, and ChihChao Fan. Optimal locations of license plate recognition to enhance the origin-destination matrix estimation. In Proceedings of the Eastern Asia Society for Transportation Studies The 9th International Conference of Eastern Asia Society for Transportation Studies, 2011, pages 297-297. Eastern Asia Society for Transportation Studies, 2011.

[116] Brett K Beaulieu-Jones and Jason H Moore. Missing data imputation in the electronic health record using deeply learned autoencoders. In Pacific Symposium on Biocomputing 2017, pages 207-218. World Scientific, 2017.

[117] Yijun Li, Sifei Liu, Jimei Yang, and Ming-Hsuan Yang. Generative face completion. In Proceedings of the IEEE Conference on Computer Vision and Pattern Recognition, pages 3911-3919, 2017.

[118] H Chang, Youngjoo Lee, B Yoon, and Sanghoon Baek. Dynamic near-term traffic flow prediction: system-oriented approach based on past experiences. IET intelligent transport systems, 6(3):292-305, 2012.

[119] Young-Seon Jeong, Young-Ji Byon, Manoel Mendonca CastroNeto, and Said M Easa. Supervised weighting-online learning algorithm for short-term traffic flow prediction. IEEE Transactions on Intelligent Transportation Systems, 14(4):1700-1707, 2013.

[120] Jun Xu, Rouhollah Rahmatizadeh, Ladislau Bölöni, and Damla Turgut. Real-time prediction of taxi demand using recurrent neural networks. IEEE Transactions on Intelligent Transportation Systems, 2017.

[121] Nan Zhang, Fei Yue Wang, Fenghua Zhu, Dongbin Zhao, and Shuming Tang. DynaCAS: Computational Experiments and
Decision Support for ITS. IEEE Intelligent Systems, 23(6):19-23, 2008.

[122] Shlomo Zeitman. Parking management system, August 171999. US Patent 5,940,481.

[123] Parisa Pouladzadeh, Pallavi Kuhad, Sri Vijay Bharat Peddi, Abdulsalam Yassine, and Shervin Shirmohammadi. Food calorie measurement using deep learning neural network. In IEEE International Instrumentation and Measurement Technology Conference Proceedings (I2MTC), pages 1-6, 2016.

[124] Chang Di Huang, Chien Yao Wang, and Jia Ching Wang. Human action recognition system for elderly and children care using three stream ConvNet. In International Conference on Orange Technologies, pages 5-9, 2016.

[125] Nils Yannick Hammerla, James Fisher, Peter Andras, Lynn Rochester, Richard Walker, and Thomas Plötz. PD disease state assessment in naturalistic environments using deep learning. In AAAI, pages 1742-1748, 2015.

[126] U Rajendra Acharya, Hamido Fujita, Shu Lih Oh, Yuki Hagiwara, Jen Hong Tan, and Muhammad Adam. Application of deep convolutional neural network for automated detection of myocardial infarction using ECG signals. Information Sciences, 415:190-198, 2017.

[127] Min Wang, Sherif Abdelfattah, Nour Moustafa, and Jiankun Hu. Deep gaussian mixture-hidden markov model for classification of eeg signals. IEEE Transactions on Emerging Topics in Computational Intelligence, 2(4):278-287, 2018.

[128] Manu Goyal, Neil D Reeves, Adrian K Davison, Satyan Rajbhandari, Jennifer Spragg, and Moi Hoon Yap. Dfunet: Convolutional neural networks for diabetic foot ulcer classification. IEEE Transactions on Emerging Topics in Computational Intelligence, 2018.

[129] Marios Anthimopoulos, Stergios Christodoulidis, Lukas Ebner, Andreas Christe, and Stavroula Mougiakakou. Lung pattern classification for interstitial lung diseases using a deep convolutional neural network. IEEE transactions on medical imaging, 35(5):12071216, 2016.

[130] MR Avendi, Arash Kheradvar, and Hamid Jafarkhani. A combined deep-learning and deformable-model approach to fully automatic segmentation of the left ventricle in cardiac MRI. Medical image analysis, 30:108-119, 2016.

[131] National Oceanic and Atmospheric Administration (NOAA). State of the science fact sheet. Technical report, United States Department of Commerce, 2016.

[132] Alex Pentland and Tanzeem Choudhury. Face recognition for smart environments. Computer, 33(2):50-55, 2000.

[133] Zhimin Cao, Qi Yin, Xiaoou Tang, and Jian Sun. Face recognition with learning-based descriptor. In 2010 IEEE Computer society conference on computer vision and pattern recognition, pages 27072714. IEEE, 2010.

[134] Omaima FathElrahman Osman and Moi Hoon Yap. Computational intelligence in automatic face age estimation: A survey. IEEE Transactions on Emerging Topics in Computational Intelligence, 1(99):1-15, 2018.

[135] Linjie Yang, Ping Luo, Chen Change Loy, and Xiaoou Tang. A large-scale car dataset for fine-grained categorization and verification. In Proceedings of the IEEE Conference on Computer Vision and Pattern Recognition, pages 3973-3981, 2015.

[136] Ashish Vaswani, Noam Shazeer, Niki Parmar, Jakob Uszkoreit, Llion Jones, Aidan N Gomez, Łukasz Kaiser, and Illia Polosukhin. Attention is all you need. In Advances in Neural Information Processing Systems, pages 6000-6010, 2017.

[137] Ian Goodfellow, Jean Pouget-Abadie, Mehdi Mirza, Bing Xu, David Warde-Farley, Sherjil Ozair, Aaron Courville, and Yoshua Bengio. Generative adversarial nets. In Advances in neural information processing systems, pages 2672-2680, 2014.

[138] Yanan Sun, Gary G Yen, and Zhang Yi. Evolving unsupervised deep neural networks for learning meaningful representations. IEEE Transactions on Evolutionary Computation, 23(1):89-103, 2019

[139] Masanori Suganuma, Shinichi Shirakawa, and Tomoharu Nagao. A genetic programming approach to designing convolutional neural network architectures. In Proceedings of the Genetic and Evolutionary Computation Conference, pages 497-504. ACM, 2017.

[140] Hanxiao Liu, Karen Simonyan, Oriol Vinyals, Chrisantha Fernando, and Koray Kavukcuoglu. Hierarchical representations for efficient architecture search. In International Conference on Learning Representations (ICLR 2018), 2017.

[141] Esteban Real, Sherry Moore, Andrew Selle, Saurabh Saxena, Yutaka Leon Suematsu, Jie Tan, Quoc V Le, and Alexey Kurakin. 
Large-scale evolution of image classifiers. In Proceedings of the 34th International Conference on Machine Learning-Volume 70, pages 2902-2911. JMLR. org, 2017.

[142] Barret Zoph and Quoc V Le. Neural architecture search with reinforcement learning. In International Conference on Learning Representations (ICLR 2017), 2016.

[143] Bowen Baker, Otkrist Gupta, Nikhil Naik, and Ramesh Raskar. Designing neural network architectures using reinforcement learning. In International Conference on Learning Representations (ICLR 2017), 2016.

[144] Vassilios S Verykios, Elisa Bertino, Igor Nai Fovino, Loredana Parasiliti Provenza, Yucel Saygin, and Yannis Theodoridis. State-of-the-art in privacy preserving data mining. ACM Sigmod Record, 33(1):50-57, 2004.

[145] Edward Choi, Siddharth Biswal, Bradley Malin, Jon Duke, Walter F Stewart, and Jimeng Sun. Generating multi-label discrete patient records using generative adversarial networks. In Machine Learning for Healthcare Conference, pages 286-305, 2017.

[146] Dan Yin and Qing Yang. Gans based density distribution privacypreservation on mobility data. Security and Communication Networks, 2018, 2018.

[147] Jen-Cheng Hou, Syu-Siang Wang, Ying-Hui Lai, Yu Tsao, HsiuWen Chang, and Hsin-Min Wang. Audio-visual speech enhancement using multimodal deep convolutional neural networks. IEEE Transactions on Emerging Topics in Computational Intelligence, 2(2):117-128, 2018.

[148] Wei Wang and Min Zhang. Tensor deep learning model for heterogeneous data fusion in internet of things. IEEE Transactions on Emerging Topics in Computational Intelligence, 2018.

[149] W. Wang, S. De, Y. Zhou, X. Huang, and K. Moessner. Distributed sensor data computing in smart city applications. In 2017 IEEE 18th International Symposium on A World of Wireless, Mobile and Multimedia Networks (WoWMoM), pages 1-5, June 2017. 